\title{
Syntheses and structures of two new lithium-heptamolybdates
}

Savita S. Khandolkar ${ }^{\mathrm{a}}$, Christian Näther ${ }^{\mathrm{b}}$, Wolfgang Bensch ${ }^{\mathrm{b}}$, Bikshandarkoil R. Srinivasan $^{\mathrm{a}}$, a'Department of Chemistry, Goa University, Goa 403206, India Email: srini@unigoa.ac.in

${ }^{\mathrm{b}}$ Institut für Anorganische Chemie, Christian-Albrechts-Universität Kiel

Max-Eyth Straße 2, D-24098 Kiel, Germany

\section{SUPPLEMENTARY MATERIAL FOR ONLINE VERSION}

Table S1. List of structurally characterized heptamolybdate compounds.

\begin{tabular}{|c|c|c|c|c|}
\hline No & Compound & $\begin{array}{l}\text { Space } \\
\text { Group }\end{array}$ & $\begin{array}{l}\text { Binding mode } \\
\text { of }\left\{\mathrm{Mo}_{7} \mathrm{O}_{24}\right\}^{-6}\end{array}$ & Ref \\
\hline 1 & $\left(\mathrm{H}_{2} \mathrm{DABCO}\right)_{3}\left[\mathrm{Mo}_{7} \mathrm{O}_{24}\right] \cdot 4 \mathrm{H}_{2} \mathrm{O}$ & $C c$ & Counterion & 26 \\
\hline 2 & $(2-\mathrm{ampH})_{4}\left[\mathrm{Co}\left(\mathrm{H}_{2} \mathrm{O}\right)_{5} \mathrm{Mo}_{7} \mathrm{O}_{24}\right] \cdot 9 \mathrm{H}_{2} \mathrm{O}$ & $\operatorname{Pna2}_{1}$ & Monodentate & 9 \\
\hline 3 & {$[3-\mathrm{ampH}]_{4}\left[\left\{\mathrm{Zn}(3-\mathrm{ampy})\left(\mathrm{H}_{2} \mathrm{O}\right)_{4}\right\} \mathrm{Mo}_{7} \mathrm{O}_{24}\right] \cdot 4 \mathrm{H}_{2} \mathrm{O}$} & $C c$ & Monodentate & 15 \\
\hline 4 & {$[3-\mathrm{ampH}]_{4}\left[\left\{\mathrm{Co}(3-\mathrm{ampy})\left(\mathrm{H}_{2} \mathrm{O}\right)_{4}\right\} \mathrm{Mo}_{7} \mathrm{O}_{24}\right] \cdot 4 \mathrm{H}_{2} \mathrm{O}$} & $C c$ & Monodentate & 15 \\
\hline 5 & {$[2-\mathrm{ampH}]_{4}\left[\left\{\mathrm{Zn}\left(\mathrm{H}_{2} \mathrm{O}\right)_{5}\right\} \mathrm{Mo}_{7} \mathrm{O}_{24}\right] \cdot 9 \mathrm{H}_{2} \mathrm{O}$} & Pnma & Monodentate & 15 \\
\hline 6 & $\mathrm{Na}\left(\mathrm{NH}_{4}\right)[\mathrm{bppH}]_{2}\left[\mathrm{Mo}_{7} \mathrm{O}_{24}\right] \cdot 8 \mathrm{H}_{2} \mathrm{O}$ & $P 2_{1} / m$ & Tridentate & 8 \\
\hline 7 & $\left(\mathrm{NH}_{4}\right)_{4}\left[\mathrm{Ru}(\mathrm{DMSO})_{3} \mathrm{Mo}_{7} \mathrm{O}_{24}\right] \cdot 6.5 \mathrm{H}_{2} \mathrm{O}$ & $C 2 / c$ & Tridentate & 14 \\
\hline 8 & $\left(\mathrm{NH}_{4}\right)_{4}\left[\mathrm{Os}(\mathrm{DMSO})_{3} \mathrm{Mo}_{7} \mathrm{O}_{24}\right] \cdot 4.5 \mathrm{H}_{2} \mathrm{O}$ & $C 2 / c$ & Tridentate & 14 \\
\hline 9 & $(\mathrm{ImH})_{4}\left[\mathrm{Ca}\left(\mathrm{H}_{2} \mathrm{O}\right)_{6}(\mu-\mathrm{O})_{2}\left\{\mathrm{Mo}_{7} \mathrm{O}_{24}\right\}\right] \cdot 2(\mathrm{Im}) \cdot 3 \mathrm{H}_{2} \mathrm{O}$ & $C 2 / m$ & $\mu_{2}$-bidentate & 17 \\
\hline 10 & $\left(\mathrm{NH}_{4}\right)_{6} \mathrm{H}_{2}\left[\mathrm{Cu}\left(\mathrm{C}_{2} \mathrm{O}_{4}\right)_{2}\left(\mathrm{Mo}_{7} \mathrm{O}_{24}\right)\right] \cdot 9 \mathrm{H}_{2} \mathrm{O}$ & $P 2{ }_{1} / m$ & $\mu_{2}$-bidentate & 18 \\
\hline 11 & $(\mathrm{hmtH})_{2}\left[\left\{\mathrm{Mg}\left(\mathrm{H}_{2} \mathrm{O}\right)_{5}\right\}_{2}\left\{\mathrm{Mo}_{7} \mathrm{O}_{24}\right\}\right] \cdot 3 \mathrm{H}_{2} \mathrm{O}$ & $C 2 / c$ & $\mu_{2}$-bidentate & 10 \\
\hline 12 & $(\mathrm{hmtH})_{2}\left[\left\{\left(\mathrm{Zn}\left(\mathrm{H}_{2} \mathrm{O}\right)_{5}\right\}\left\{\mathrm{Zn}\left(\mathrm{H}_{2} \mathrm{O}\right)_{4}\right\}\left\{\mathrm{Mo}_{7} \mathrm{O}_{24}\right\}\right] \cdot 2 \mathrm{H}_{2} \mathrm{O}\right.$ & $C 2 / c$ & $\mu_{2}$-tridentate & 11 \\
\hline 13 & $(\mathrm{hmtH})_{2}\left[\mathrm{Mn}_{2}\left(\mathrm{H}_{2} \mathrm{O}\right)_{9} \mathrm{Mo}_{7} \mathrm{O}_{24}\right] \cdot 2 \mathrm{H}_{2} \mathrm{O}$ & $C 2 / c$ & $\mu_{2}$-tridentate & 12 \\
\hline 14 & $(\mathrm{hmtH})_{2}\left[\mathrm{Fe}_{2}\left(\mathrm{H}_{2} \mathrm{O}\right)_{9} \mathrm{Mo}_{7} \mathrm{O}_{24}\right] \cdot 2 \mathrm{H}_{2} \mathrm{O}$ & $C 2 / c$ & $\mu_{2}$-tridentate & 12 \\
\hline 15 & $\left(\mathrm{NH}_{4}\right)\left[\mathrm{Cu}(\mathrm{en})_{2}\right]\left[\mathrm{Na}(\mathrm{en}) \mathrm{Cu}(\mathrm{en})_{2}\left(\mathrm{H}_{2} \mathrm{O}\right)\left(\mathrm{Mo}_{7} \mathrm{O}_{24}\right)\right] \cdot 4 \mathrm{H}_{2} \mathrm{O}$ & $P_{1}^{-}$ & $\mu_{3}$-tridentate & 19 \\
\hline 16 & $\left(\mathrm{GuaNH}_{2}\right)_{7} \mathrm{Na}\left[\mathrm{Co}\left(\mathrm{H}_{2} \mathrm{O}\right)_{5}\left\{\mathrm{Mo}_{7} \mathrm{O}_{24}\right\}\right]_{2} \cdot 8 \mathrm{H}_{2} \mathrm{O}$ & $P 2_{1} / c$ & $\mu_{2}$-tetradentate & 13 \\
\hline 17 & {$\left[\left(\mathrm{CH}_{3}\right)_{4} \mathrm{~N}\right]_{1.33}\left[\mathrm{NH}_{4}\right]_{12.67}\left[\mathrm{La}_{4}\left(\mathrm{MoO}_{4}\right)\left(\mathrm{H}_{2} \mathrm{O}\right)_{16}\left(\mathrm{Mo}_{7} \mathrm{O}_{24}\right)_{4}\right] \cdot 12 \mathrm{H}_{2} \mathrm{O}$} & $I_{4}^{-} 3 d$ & $\mu_{3}$-tetradentate & 21 \\
\hline 18 & {$\left[\mathrm{NH}_{4}\right]_{14}\left[\mathrm{Ce}_{4}\left(\mathrm{MoO}_{4}\right)\left(\mathrm{H}_{2} \mathrm{O}\right)_{16}\left(\mathrm{Mo}_{7} \mathrm{O}_{24}\right)_{4}\right] \cdot 36 \mathrm{H}_{2} \mathrm{O}$} & $P_{1}^{-}$ & $\mu_{3}$-tetradentate & 21 \\
\hline 19 & {$\left[\mathrm{NH}_{4}\right]_{14}\left[\mathrm{Pr}_{4}\left(\mathrm{MoO}_{4}\right)\left(\mathrm{H}_{2} \mathrm{O}\right)_{16}\left(\mathrm{Mo}_{7} \mathrm{O}_{24}\right)_{4}\right] \cdot 29 \mathrm{H}_{2} \mathrm{O}$} & $C 2 / c$ & $\mu_{3}$-tetradentate & 21 \\
\hline 20 & {$\left[\left(\mathrm{CH}_{3}\right)_{4} \mathrm{~N}\right]_{3.33}\left[\mathrm{NH}_{4}\right]_{10.67}\left[\mathrm{Sm}_{4}\left(\mathrm{MoO}_{4}\right)\left(\mathrm{H}_{2} \mathrm{O}\right)_{16}\left(\mathrm{Mo}_{7} \mathrm{O}_{24}\right)_{4}\right] \cdot 22 \mathrm{H}_{2} \mathrm{O}$} & $I_{\overline{4}}^{\overline{4}} 3 d$ & $\mu_{3}$-tetradentate & 21 \\
\hline 21 & {$\left[\left(\mathrm{CH}_{3}\right)_{4} \mathrm{~N}\right]_{3.33}\left[\mathrm{NH}_{4}\right]_{10.67}\left[\mathrm{Gd}_{4}\left(\mathrm{MoO}_{4}\right)\left(\mathrm{H}_{2} \mathrm{O}\right)_{16}\left(\mathrm{Mo}_{7} \mathrm{O}_{24}\right)_{4}\right] \cdot 16 \mathrm{H}_{2} \mathrm{O}$} & $I_{\overline{4}} 3 d$ & $\mu_{3}$-tetradentate & 21 \\
\hline 22 & $\mathrm{Na}_{2}\left(\mathrm{hmtH}_{2}\right)_{2}\left[\mathrm{Mo}_{7} \mathrm{O}_{24}\right] \cdot 9 \mathrm{H}_{2} \mathrm{O}$ & Pnma & $\mu_{2}$-pentadentate & 16 \\
\hline 23 & $\left.\left[(\mathrm{hmtH})_{2}\right\}_{1.5}\left\{(\mathrm{ahmtH})_{2}\right\}_{0.5}\right]\left[\mathrm{Na}_{2}\left(\mathrm{H}_{2} \mathrm{O}\right)_{5} \mathrm{Mo}_{7} \mathrm{O}_{24}\right] \cdot 4 \mathrm{H}_{2} \mathrm{O}$ & Pnma & $\mu_{2}$-pentadentate & 12 \\
\hline 24 & $\left(\mathrm{NH}_{4}\right)_{28}\left[\mathrm{Ce}_{8}\left(\mathrm{MoO}_{4}\right)_{2}\left(\mathrm{H}_{2} \mathrm{O}\right)_{31}\left(\mathrm{Mo}_{7} \mathrm{O}_{24}\right)_{8}\right] \cdot 74 \mathrm{H}_{2} \mathrm{O}$ & $P_{1}^{-}$ & $\begin{array}{l}\mu_{3} \text {-tetradentate, } \\
\mu_{4} \text {-pentadentate }\end{array}$ & 20 \\
\hline 25 & $\left(\mathrm{NH}_{4}\right)_{26}\left[\mathrm{CoPr}_{8}\left(\mathrm{MoO}_{4}\right)_{2}\left(\mathrm{H}_{2} \mathrm{O}\right)_{33}\left(\mathrm{Mo}_{7} \mathrm{O}_{24}\right)_{8}\right] \cdot 54 \mathrm{H}_{2} \mathrm{O}$ & $P_{\overline{1}}^{-}$ & $\begin{array}{l}\mu_{3} \text {-tetradentate, } \\
\mu_{4} \text {-pentadentate, } \\
\mu_{4} \text {-hexadentate }\end{array}$ & 20 \\
\hline 26 & $\left(\mathrm{NH}_{4}\right)_{11.9}\left[\mathrm{Nd}_{4.7}\left(\mathrm{MoO}_{4}\right)\left(\mathrm{H}_{2} \mathrm{O}\right)_{23}\left(\mathrm{Mo}_{7} \mathrm{O}_{24}\right)_{4}\right] \cdot 19 \mathrm{H}_{2} \mathrm{O}$ & $P \overline{1}$ & $\begin{array}{l}\mu_{3} \text {-tetradentate, } \\
\mu_{4} \text {-hexadentate }\end{array}$ & 20 \\
\hline 27 & $\left(\mathrm{NH}_{4}\right)_{11.9}\left[\operatorname{Pr}_{4.7}\left(\mathrm{MoO}_{4}\right)\left(\mathrm{H}_{2} \mathrm{O}\right)_{23}\left(\mathrm{Mo}_{7} \mathrm{O}_{24}\right)_{4}\right] \cdot 34 \mathrm{H}_{2} \mathrm{O}$ & $P \overline{1}$ & $\begin{array}{l}\mu_{3} \text {-tetradentate, } \\
\mu_{4} \text {-hexadentate }\end{array}$ & 20 \\
\hline 28 & $\mathrm{Na}_{7}\left[\mathrm{Mo}_{7} \mathrm{O}_{24}\right](\mathrm{OH}) \cdot 21 \mathrm{H}_{2} \mathrm{O}$ & $P 2 / n$ & $\mu_{6}$-hexadentate & 7 \\
\hline 29 & $\mathrm{Na}_{6}\left[\mathrm{Mo}_{7} \mathrm{O}_{24}\right] \cdot 14 \mathrm{H}_{2} \mathrm{O}$ & $\mathrm{Pca}_{1}$ & $\mu_{11}$-pentadecadentate & 5 \\
\hline 30 & $\mathrm{NaCs}_{5}\left[\mathrm{Mo}_{7} \mathrm{O}_{24}\right] \cdot 5 \mathrm{H}_{2} \mathrm{O}$ & $P{ }_{1}^{-}$ & $\mu_{16}$ hentriacontadentate & 25 \\
\hline 31 & $\mathrm{Cs}_{6}\left[\mathrm{Mo}_{7} \mathrm{O}_{24}\right] \cdot 7 \mathrm{H}_{2} \mathrm{O}$ & $P{ }_{1}^{1}$ & $\mu_{18}$-tritetracontadentate & 6 \\
\hline 32 & $\mathrm{~K}_{6}\left[\mathrm{Mo}_{7} \mathrm{O}_{24}\right] \cdot 4 \mathrm{H}_{2} \mathrm{O}$ & $P 2_{1} / c$ & --- & 23 \\
\hline 33 & $\left(\mathrm{NH}_{4}\right)_{3}\left[\mathrm{Li}_{3}\left(\mathrm{H}_{2} \mathrm{O}\right)_{4}\left(\mu_{6}-\mathrm{Mo}_{7} \mathrm{O}_{24}\right\}\right] \cdot 2 \mathrm{H}_{2} \mathrm{O} 2$ & Pnma & $\mu_{6}$-hexadentate & This work \\
\hline 34 & $\left(\mathrm{NH}_{4}\right)_{4}\left[\mathrm{Li}_{2}\left(\mathrm{H}_{2} \mathrm{O}\right)_{7}\right]\left[\mathrm{Mo}_{7} \mathrm{O}_{24}\right] \cdot \mathrm{H}_{2} \mathrm{O} 1$ & $P 2_{1} / n$ & Counterion & This work \\
\hline
\end{tabular}




\begin{tabular}{|c|c|c|c|c|}
\hline 35 & $\left(\mathrm{NH}_{4}\right)_{6}\left[\mathrm{Mo}_{7} \mathrm{O}_{24}\right] \cdot 4 \mathrm{H}_{2} \mathrm{O}$ & $P 2{ }_{l} / c$ & Counterion & 22,23 \\
\hline 36 & $(2-\mathrm{ampH})_{6}\left[\mathrm{Mo}_{7} \mathrm{O}_{24}\right] \cdot 3 \mathrm{H}_{2} \mathrm{O}$ & $P 2_{1} / n$ & Counterion & 24 \\
\hline 37 & $\left(\mathrm{BuNH}_{3}\right)_{8}\left[\left(\mathrm{Mo}_{7} \mathrm{O}_{24}\right)\left(\mathrm{MoO}_{4}\right)\right] \cdot 3 \mathrm{H}_{2} \mathrm{O}$ & $P_{1}^{-}$ & Counterion & 4 \\
\hline 38 & $\left(\mathrm{BuNH}_{3}\right)_{6}\left[\mathrm{Mo}_{7} \mathrm{O}_{24}\right] \cdot 4 \mathrm{H}_{2} \mathrm{O}$ & $P 2_{1} / c$ & Counterion & To be published \\
\hline 39 & $\left(\mathrm{BuNH}_{3}\right)_{6}\left[\mathrm{Mo}_{7} \mathrm{O}_{24}\right] \cdot 3 \mathrm{H}_{2} \mathrm{O}$ & $P 2{ }_{1} / n$ & Counterion & 27 \\
\hline 40 & $\left(\mathrm{PyrNH}_{3}\right)_{6}\left[\left(\mathrm{Mo}_{7} \mathrm{O}_{24}\right] \cdot 2 \mathrm{H}_{2} \mathrm{O}\right.$ & $P_{1}^{-}$ & Counterion & 4 \\
\hline 41 & $\left(\mathrm{PrNH}_{3}\right)_{6}\left[\mathrm{Mo}_{7} \mathrm{O}_{24}\right] \cdot 3 \mathrm{H}_{2} \mathrm{O}$ & $P_{1}^{-}$ & Counterion & 4,27 \\
\hline 42 & $\left(\mathrm{PentNH}_{3}\right)_{6}\left[\mathrm{Mo}_{7} \mathrm{O}_{24}\right] \cdot 3 \mathrm{H}_{2} \mathrm{O}$ & $P 2_{1} / n$ & Counterion & 4,27 \\
\hline 43 & $\left(\mathrm{HexNH}_{3}\right)_{6}\left[\mathrm{Mo}_{7} \mathrm{O}_{24}\right] \cdot 3 \mathrm{H}_{2} \mathrm{O}$ & $P 2_{1} / n$ & Counterion & 27 \\
\hline 44 & $\left(\mathrm{t}-\mathrm{BuNH}_{3}\right)_{6}\left[\mathrm{Mo}_{7} \mathrm{O}_{24}\right] \cdot 7 \mathrm{H}_{2} \mathrm{O}$ & $P 2_{1} / n$ & Counterion & 28 \\
\hline 45 & $\left(\text { TemedH }{ }_{2}\right)_{3}\left[\mathrm{Mo}_{7} \mathrm{O}_{24}\right] \cdot 4 \mathrm{H}_{2} \mathrm{O}$ & $C 2 / c$ & Counterion & 29 \\
\hline 46 & $\left(\mathrm{GuaNH}_{2}\right)_{6}\left[\mathrm{Mo}_{7} \mathrm{O}_{24}\right] \cdot \mathrm{H}_{2} \mathrm{O}$ & $C 2 / c$ & Counterion & 30 \\
\hline 47 & $\left(\mathrm{GuaNH}_{2}\right)_{6}\left[\mathrm{Mo}_{7} \mathrm{O}_{24}\right] \cdot \mathrm{H}_{2} \mathrm{O}$ & $P 2{ }_{1} / c$ & Counterion & 31 \\
\hline 48 & $(4-\mathrm{apH})_{6}\left[\mathrm{Mo}_{7} \mathrm{O}_{24}\right] \cdot 6 \mathrm{H}_{2} \mathrm{O}$ & $P 2{ }_{1} / c$ & Counterion & 32 \\
\hline 49 & {$[\mathrm{UreaH}]_{3}\left(\mathrm{NH}_{4}\right)_{9}\left[\mathrm{Mo}_{7} \mathrm{O}_{24}\right]_{2} \cdot 5[$ Urea $] \cdot 4 \mathrm{H}_{2} \mathrm{O}$} & Fddd & Counterion & 17 \\
\hline 50 & $\left(\operatorname{dienH} H_{3}\right)_{2}\left[\mathrm{Mo}_{7} \mathrm{O}_{24}\right] \cdot 4 \mathrm{H}_{2} \mathrm{O}$ & $C 2 / c$ & Counterion & 33 \\
\hline 51 & $\left(\operatorname{dienH} H_{3}\right)_{2}\left[\mathrm{Mo}_{7} \mathrm{O}_{24}\right] \cdot 4 \mathrm{H}_{2} \mathrm{O}$ & $P 2{ }_{1} / a$ & Counterion & 33 \\
\hline 52 & $\left(\mathrm{NMe}_{4}\right)_{2}\left(\mathrm{NH}_{4}\right)_{8}\left[\left(\mathrm{Mo}_{7} \mathrm{O}_{22}\right)\left(\mu_{2}-\mathrm{O}\right)_{2}\left(\mathrm{Mo}_{7} \mathrm{O}_{22}\right)\right] \cdot 4 \mathrm{H}_{2} \mathrm{O}$ & $P_{1}^{-}$ & Counterion & 34 \\
\hline 53 & $\left(\mathrm{BuNH}_{3}\right)_{10}\left[\left(\mathrm{Mo}_{7} \mathrm{O}_{22}\right)\left(\mu_{2}-\mathrm{O}\right)_{2}\left(\mathrm{Mo}_{7} \mathrm{O}_{22}\right)\right] \cdot 5.5 \mathrm{H}_{2} \mathrm{O}$ & $P 2_{1} / n$ & Counterion & 4 \\
\hline 54 & {$[2,3-\operatorname{diampH}]_{4}\left[\mathrm{Co}\left(\mathrm{H}_{2} \mathrm{O}\right)_{6}\right]\left[\mathrm{Mo}_{7} \mathrm{O}_{24}\right] \cdot 6 \mathrm{H}_{2} \mathrm{O}$} & $C 2 / c$ & Counterion & 15 \\
\hline
\end{tabular}

Table S2. Metric parameters $\left(\AA{ }^{\circ}{ }^{\circ}\right)$ of $\left(\mathrm{Mo}_{7} \mathrm{O}_{24}\right)^{6-}$ unit in $\left(\mathrm{NH}_{4}\right)_{4}\left[\mathrm{Li}_{2}\left(\mathrm{H}_{2} \mathrm{O}\right)_{7}\right]\left[\mathrm{Mo}_{7} \mathrm{O}_{24}\right] \cdot \mathrm{H}_{2} \mathrm{O} \mathbf{1}$.

\section{Bond lengths}

$\begin{array}{llll}\text { Mo(1)-O(2) } & 1.721(3) & \mathrm{Mo}(4)-\mathrm{O}(20) & 1.897(3) \\ \mathrm{Mo}(1)-\mathrm{O}(4) & 1.733(3) & \mathrm{Mo}(4)-\mathrm{O}(5) & 1.899(3) \\ \mathrm{Mo}(1)-\mathrm{O}(6) & 1.931(3) & \mathrm{Mo}(4)-\mathrm{O}(3) & 2.267(3) \\ \mathrm{Mo}(1)-\mathrm{O}(1) & 1.990(3) & \mathrm{Mo}(4)-\mathrm{O}(8) & 2.282(3) \\ \mathrm{Mo}(1)-\mathrm{O}(3) & 2.158(3) & \mathrm{Mo}(5)-\mathrm{O}(16) & 1.722(3) \\ \mathrm{Mo}(1)-\mathrm{O}(5) & 2.244(3) & \mathrm{Mo}(5)-\mathrm{O}(15) & 1.742(3) \\ \mathrm{Mo}(1)-\mathrm{Mo}(3) & 3.2110(5) & \mathrm{Mo}(5)-\mathrm{O}(10) & 1.913(3) \\ \mathrm{Mo}(2)-\mathrm{O}(7) & 1.713(3) & \mathrm{Mo}(5)-\mathrm{O}(23) & 1.917(3) \\ \mathrm{Mo}(2)-\mathrm{O}(9) & 1.721(3) & \mathrm{Mo}(5)-\mathrm{O}(8) & 2.152(3) \\ \mathrm{Mo}(2)-\mathrm{O}(6) & 1.944(3) & \mathrm{Mo}(6)-\mathrm{O}(18) & 1.716(3) \\ \mathrm{Mo}(2)-\mathrm{O}(10) & 1.991(3) & \mathrm{Mo}(6)-\mathrm{O}(19) & 1.737(3) \\ \mathrm{Mo}(2)-\mathrm{O}(8) & 2.149(3) & \mathrm{Mo}(6)-\mathrm{O}(21) & 1.936(3) \\ \mathrm{Mo}(2)-\mathrm{O}(5) & 2.284(3) & \mathrm{Mo}(6)-\mathrm{O}(17) & 1.949(3) \\ \mathrm{Mo}(3)-\mathrm{O}(11) & 1.722(3) & \mathrm{Mo}(6)-\mathrm{O}(3) & 2.156(3) \\ \mathrm{Mo}(3)-\mathrm{O}(12) & 1.728(3) & \mathrm{Mo}(6)-\mathrm{O}(20) & 2.272(3) \\ \mathrm{Mo}(3)-\mathrm{O}(17) & 1.924(3) & \mathrm{Mo}(7)-\mathrm{O}(24) & 1.706(3) \\ \mathrm{Mo}(3)-\mathrm{O}(1) & 1.926(3) & \mathrm{Mo}(7)-\mathrm{O}(22) & 1.730(3) \\ \mathrm{Mo}(3)-\mathrm{O}(3) & 2.159(3) & \mathrm{Mo}(7)-\mathrm{O}(21) & 1.929(3) \\ \mathrm{Mo}(3)-\mathrm{O}(11) & 1.722(3) & \mathrm{Mo}(7)-\mathrm{O}(23) & 1.985(3) \\ \mathrm{Mo}(4)-\mathrm{O}(14) & 1.740(3) & \mathrm{Mo}(7)-\mathrm{O}(8) & 2.146(3) \\ \mathrm{Mo}(4)-\mathrm{O}(13) & 1.757(3) & \mathrm{Mo}(7)-\mathrm{O}(20) & 2.277(3)\end{array}$

\section{Bond angles}

$\begin{array}{llll}\mathrm{O}(2)-\mathrm{Mo}(1)-\mathrm{O}(4) & 105.19(17) & \mathrm{O}(16)-\mathrm{Mo}(5)-\mathrm{O}(23) & 97.74(15) \\ \mathrm{O}(2)-\mathrm{Mo}(1)-\mathrm{O}(6) & 96.99(15) & \mathrm{O}(15)-\mathrm{Mo}(5)-\mathrm{O}(23) & 102.34(15) \\ \mathrm{O}(4)-\mathrm{Mo}(1)-\mathrm{O}(6) & 100.94(15) & \mathrm{O}(10)-\mathrm{Mo}(5)-\mathrm{O}(23) & 147.09(13) \\ \mathrm{O}(2)-\mathrm{Mo}(1)-\mathrm{O}(1) & 98.83(15) & \mathrm{O}(16)-\mathrm{Mo}(5)-\mathrm{O}(8) & 106.04(14) \\ \mathrm{O}(4)-\mathrm{Mo}(1)-\mathrm{O}(1) & 92.52(15) & \mathrm{O}(15)-\mathrm{Mo}(5)-\mathrm{O}(8) & 147.79(14)\end{array}$




\begin{tabular}{|c|c|c|c|}
\hline $\mathrm{O}(6)-\mathrm{Mo}(1)-\mathrm{O}(1)$ & $155.75(14)$ & $\mathrm{O}(10)-\mathrm{Mo}(5)-\mathrm{O}(8)$ & $74.14(12)$ \\
\hline $\mathrm{O}(2)-\mathrm{Mo}(1)-\mathrm{O}(3)$ & $95.73(14)$ & $\mathrm{O}(23)-\mathrm{Mo}(5)-\mathrm{O}(8)$ & $73.95(12)$ \\
\hline $\mathrm{O}(4)-\mathrm{Mo}(1)-\mathrm{O}(3)$ & $156.38(15)$ & $\mathrm{O}(18)-\mathrm{Mo}(6)-\mathrm{O}(19)$ & $104.19(17)$ \\
\hline $\mathrm{O}(6)-\mathrm{Mo}(1)-\mathrm{O}(3)$ & $87.01(12)$ & $\mathrm{O}(18)-\mathrm{Mo}(6)-\mathrm{O}(21)$ & $96.72(14)$ \\
\hline $\mathrm{O}(1)-\mathrm{Mo}(1)-\mathrm{O}(3)$ & $73.24(12)$ & $\mathrm{O}(19)-\mathrm{Mo}(6)-\mathrm{O}(21)$ & $98.90(14)$ \\
\hline $\mathrm{O}(2)-\mathrm{Mo}(1)-\mathrm{O}(5)$ & $165.72(14)$ & $\mathrm{O}(18)-\mathrm{Mo}(6)-\mathrm{O}(17)$ & $100.86(15)$ \\
\hline $\mathrm{O}(4)-\mathrm{Mo}(1)-\mathrm{O}(5)$ & $87.96(14)$ & $\mathrm{O}(19)-\mathrm{Mo}(6)-\mathrm{O}(17)$ & $94.05(14)$ \\
\hline $\mathrm{O}(6)-\mathrm{Mo}(1)-\mathrm{O}(5)$ & $74.66(12)$ & $\mathrm{O}(21)-\mathrm{Mo}(6)-\mathrm{O}(17)$ & $154.88(13)$ \\
\hline $\mathrm{O}(1)-\mathrm{Mo}(1)-\mathrm{O}(5)$ & $85.88(13)$ & $\mathrm{O}(18)-\mathrm{Mo}(6)-\mathrm{O}(3)$ & $95.14(14)$ \\
\hline $\mathrm{O}(3)-\mathrm{Mo}(1)-\mathrm{O}(5)$ & $72.60(11)$ & $\mathrm{O}(19)-\mathrm{Mo}(6)-\mathrm{O}(3)$ & $158.76(14)$ \\
\hline $\mathrm{O}(7)-\mathrm{Mo}(2)-\mathrm{O}(9)$ & $105.20(16)$ & $\mathrm{O}(21)-\mathrm{Mo}(6)-\mathrm{O}(3)$ & $87.32(12)$ \\
\hline $\mathrm{O}(7)-\mathrm{Mo}(2)-\mathrm{O}(6)$ & $100.42(15)$ & $\mathrm{O}(17)-\mathrm{Mo}(6)-\mathrm{O}(3)$ & 73.43(11) \\
\hline $\mathrm{O}(9)-\mathrm{Mo}(2)-\mathrm{O}(6)$ & $97.25(14)$ & $\mathrm{O}(18)-\mathrm{Mo}(6)-\mathrm{O}(20)$ & $164.12(14)$ \\
\hline $\mathrm{O}(7)-\mathrm{Mo}(2)-\mathrm{O}(10)$ & $91.16(15)$ & $\mathrm{O}(20)-\mathrm{Mo}(4)-\mathrm{O}(3)$ & $77.29(12)$ \\
\hline $\mathrm{O}(9)-\mathrm{Mo}(2)-\mathrm{O}(10)$ & $101.09(15)$ & $\mathrm{O}(5)-\mathrm{Mo}(4)-\mathrm{O}(3)$ & $76.90(12)$ \\
\hline $\mathrm{O}(6)-\mathrm{Mo}(2)-\mathrm{O}(10)$ & $154.92(13)$ & $\mathrm{O}(14)-\mathrm{Mo}(4)-\mathrm{O}(8)$ & \\
\hline $\mathrm{O}(7)-\mathrm{Mo}(2)-\mathrm{O}(8)$ & $155.21(14)$ & $\mathrm{O}(13)-\mathrm{Mo}(4)-\mathrm{O}(8)$ & $171.88(13)$ \\
\hline $\mathrm{O}(9)-\mathrm{Mo}(2)-\mathrm{O}(8)$ & $96.50(14)$ & $\mathrm{O}(20)-\mathrm{Mo}(4)-\mathrm{O}(8)$ & $76.92(12)$ \\
\hline $\mathrm{O}(6)-\mathrm{Mo}(2)-\mathrm{O}(8)$ & $88.38(12)$ & $\mathrm{Mo}(4)-\mathrm{O}(8)$ & 75.98 \\
\hline $\mathrm{O}(10)-\mathrm{Mo}(2)-\mathrm{O}(8)$ & $72.74(12)$ & $\mathrm{O}(3)-\mathrm{Mo}(4)-\mathrm{O}(8)$ & $88.30(10)$ \\
\hline $\mathrm{O}(7)-\mathrm{Mo}(2)-\mathrm{O}(5)$ & $88.73(14)$ & $\mathrm{O}(16)-\mathrm{Mo}(5)-\mathrm{O}(15)$ & $106.17(17)$ \\
\hline $\mathrm{O}(9)-\mathrm{Mo}(2)-\mathrm{O}(5)$ & $164.66(14)$ & $\mathrm{O}(5)-\mathrm{O}(10)$ & $98.55(15)$ \\
\hline $\mathrm{O}(6)-\mathrm{Mo}(2)-\mathrm{O}(5)$ & $73.49(12)$ & $\mathrm{O}(15)-\mathrm{Mo}(5)-\mathrm{O}(10)$ & $100.28(15)$ \\
\hline $\mathrm{O}(10)-\mathrm{Mo}(2)-\mathrm{O}(5)$ & $84.72(12)$ & $\mathrm{O}(19)-\mathrm{Mo}(6)-\mathrm{O}(20)$ & $89.86(14)$ \\
\hline $\mathrm{O}(8)-\mathrm{Mo}(2)-\mathrm{O}(5)$ & $71.48(11)$ & $\mathrm{O}(21)-\mathrm{Mo}(6)-\mathrm{O}(20)$ & $73.45(12)$ \\
\hline $\mathrm{O}(11)-\mathrm{Mo}(3)-\mathrm{O}(12)$ & 105.71(16) & $\mathrm{O}(17)-\mathrm{Mo}(6)-\mathrm{O}(20)$ & $85.22(12)$ \\
\hline $\mathrm{O}(11)-\mathrm{Mo}(3)-\mathrm{O}(17)$ & $99.50(15)$ & $\mathrm{O}(3)-\mathrm{Mo}(6)-\mathrm{O}(20)$ & $72.37(11)$ \\
\hline $\mathrm{O}(12)-\mathrm{Mo}(3)-\mathrm{O}(17)$ & $97.60(15)$ & $\mathrm{O}(24)-\mathrm{Mo}(7)-\mathrm{O}(22)$ & $105.35(17)$ \\
\hline $\mathrm{O}(11)-\mathrm{Mo}(3)-\mathrm{O}(1)$ & $98.17(16)$ & $\mathrm{O}(24)-\mathrm{Mo}(7)-\mathrm{O}(21)$ & $97.53(15)$ \\
\hline $\mathrm{O}(12)-\mathrm{Mo}(3)-\mathrm{O}(1)$ & $104.64(15)$ & $\mathrm{O}(22)-\mathrm{Mo}(7)-\mathrm{O}(21)$ & $100.14(15)$ \\
\hline $\mathrm{O}(17)-\mathrm{Mo}(3)-\mathrm{O}(1)$ & $146.53(13)$ & $\mathrm{O}(24)-\mathrm{Mo}(7)-\mathrm{O}(23)$ & $100.38(15)$ \\
\hline $\mathrm{O}(11)-\mathrm{Mo}(3)-\mathrm{O}(3)$ & 104.01(14) & $\mathrm{O}(22)-\mathrm{Mo}(7)-\mathrm{O}(23)$ & 92.72(15) \\
\hline $\mathrm{O}(12)-\mathrm{Mo}(3)-\mathrm{O}(3)$ & $150.05(15)$ & $\mathrm{O}(21)-\mathrm{Mo}(7)-\mathrm{O}(23)$ & $154.31(13)$ \\
\hline $\mathrm{O}(17)-\mathrm{Mo}(3)-\mathrm{O}(3)$ & $73.83(12)$ & $\mathrm{O}(24)-\mathrm{Mo}(7)-\mathrm{O}(8)$ & $93.34(14)$ \\
\hline $\mathrm{O}(1)-\mathrm{Mo}(3)-\mathrm{O}(3)$ & $74.43(12)$ & $\mathrm{O}(22)-\mathrm{Mo}(7)-\mathrm{O}(8)$ & $158.30(14)$ \\
\hline $\mathrm{O}(14)-\mathrm{Mo}(4)-\mathrm{O}(13)$ & $105.19(16)$ & $\mathrm{O}(21)-\mathrm{Mo}(7)-\mathrm{O}(8)$ & $87.99(12)$ \\
\hline $\mathrm{O}(14)-\mathrm{Mo}(4)-\mathrm{O}(20)$ & $100.26(15)$ & $\mathrm{O}(23)-\mathrm{Mo}(7)-\mathrm{O}(8)$ & $72.78(12)$ \\
\hline $\mathrm{O}(13)-\mathrm{Mo}(4)-\mathrm{O}(20)$ & $101.77(15)$ & $\mathrm{O}(24)-\mathrm{Mo}(7)-\mathrm{O}(20)$ & $163.13(14)$ \\
\hline $\mathrm{O}(14)-\mathrm{Mo}(4)-\mathrm{O}(5)$ & $101.16(15)$ & $\mathrm{O}(22)-\mathrm{Mo}(7)-\mathrm{O}(20)$ & $90.47(14)$ \\
\hline $\mathrm{O}(13)-\mathrm{Mo}(4)-\mathrm{O}(5)$ & $101.48(15)$ & $\mathrm{O}(21)-\mathrm{Mo}(7)-\mathrm{O}(20)$ & $73.46(12)$ \\
\hline $\mathrm{O}(20)-\mathrm{Mo}(4)-\mathrm{O}(5)$ & $142.80(13)$ & $\mathrm{O}(23)-\mathrm{Mo}(7)-\mathrm{O}(20)$ & $84.37(12)$ \\
\hline $\mathrm{O}(14)-\mathrm{Mo}(4)-\mathrm{O}(3)$ & $171.20(13)$ & $\mathrm{O}(8)-\mathrm{Mo}(7)-\mathrm{O}(20)$ & $72.47(11)$ \\
\hline $\mathrm{O}(13)-\mathrm{Mo}(4)-\mathrm{O}(3)$ & $83.60(13)$ & & \\
\hline
\end{tabular}


Table S3. Geometric parameters of the $\mathrm{O}-\mathrm{H} \cdots \mathrm{O}, \mathrm{O}-\mathrm{H} \cdots \mathrm{N}$ and $\mathrm{N}-\mathrm{H} \cdots \mathrm{O}$ hydrogen bonds length $(\AA)$ and $\left({ }^{\circ}\right)$ in the crystal structure of $\left(\mathrm{NH}_{4}\right)_{4}\left[\mathrm{Li}_{2}\left(\mathrm{H}_{2} \mathrm{O}\right)_{7}\right]\left[\mathrm{Mo}_{7} \mathrm{O}_{24}\right] \cdot \mathrm{H}_{2} \mathrm{O} \mathbf{1}$.

\begin{tabular}{|c|c|c|c|c|c|c|}
\hline D-H & $d(D-H)$ & $\mathrm{d}(\mathrm{H} \cdots \mathrm{A})$ & $<\mathrm{DHA}$ & $d(D \cdots A)$ & A & Symmetry codes \\
\hline O31-H1O & 0.850 & 1.948 & 156.47 & 2.749 & $\mathrm{O} 4$ & {$[x-1 / 2,-y+3 / 2, z+1 / 2]$} \\
\hline O32-H3O & 0.850 & 1.865 & 163.66 & 2.691 & $\mathrm{O} 6$ & {$[-\mathrm{x}+3 / 2, \mathrm{y}-1 / 2,-\mathrm{z}+3 / 2]$} \\
\hline $\mathrm{O} 32-\mathrm{H} 4 \mathrm{O}$ & 0.850 & 1.989 & 145.03 & 2.729 & $\mathrm{O} 22$ & \\
\hline O34-H7O & 0.850 & 2.096 & 147.56 & 2.850 & $\mathrm{O} 18$ & {$[-\mathrm{x}+1 / 2, \mathrm{y}-1 / 2,-\mathrm{z}+3 / 2]$} \\
\hline O34-H8O & 0.850 & 2.148 & 153.73 & 2.934 & O9 & {$[-x+3 / 2, y-1 / 2,-z+3 / 2]$} \\
\hline O35-H9O & 0.850 & 2.009 & 147.14 & 2.762 & O5 & {$[-\mathrm{x}+1,-\mathrm{y}+1,-\mathrm{z}+1]$} \\
\hline $\mathrm{O} 35-\mathrm{H} 10 \mathrm{O}$ & 0.850 & 2.355 & 2.896 & O15 & & {$[\mathrm{x}-1, \mathrm{y}, \mathrm{z}]$} \\
\hline O36-H11O & 0.850 & 2.029 & 161.32 & 2.847 & $\mathrm{O} 13$ & {$[-\mathrm{x}+1,-\mathrm{y}+1,-\mathrm{z}+1]$} \\
\hline $\mathrm{O} 36-\mathrm{H} 12 \mathrm{O}$ & 0.850 & 2.196 & 130.31 & 2.821 & $\mathrm{O} 20$ & \\
\hline O37-H13O & 0.850 & 2.023 & 168.83 & 2.861 & O9 & {$[-\mathrm{x}+3 / 2, \mathrm{y}-1 / 2,-\mathrm{z}+3 / 2]$} \\
\hline O37-H14O & 0.850 & 2.205 & 144.83 & 2.941 & N3 & \\
\hline N1-H1N1 & 0.900 & 2.222 & 155.45 & 3.064 & O16 & \\
\hline N1-H2N1 & 0.900 & 2.075 & 160.67 & 2.939 & $\mathrm{O} 2$ & {$[-\mathrm{x}+3 / 2, \mathrm{y}-1 / 2,-\mathrm{z}+3 / 2]$} \\
\hline N1-H3N1 & 0.900 & 2.340 & 174.59 & 3.237 & $\mathrm{O} 1$ & {$[\mathrm{x}+1 / 2,-\mathrm{y}+3 / 2, \mathrm{z}+1 / 2]$} \\
\hline N1-H4N1 & 0.900 & 2.209 & 137.86 & 2.939 & O11 & {$[-\mathrm{x}+3 / 2, \mathrm{y}-1 / 2,-\mathrm{z}+3 / 2]$} \\
\hline $\mathrm{N} 2-\mathrm{H} 2 \mathrm{~N} 2$ & 0.900 & 2.394 & 123.46 & 2.986 & $\mathrm{O} 4$ & \\
\hline $\mathrm{N} 2-\mathrm{H} 2 \mathrm{~N} 2$ & 0.900 & 2.623 & 136.08 & 3.330 & $\mathrm{O} 35$ & {$[-x+1,-y+1,-z+1]$} \\
\hline N2-H3N2 & 0.900 & 2.280 & 143.19 & 3.049 & O15 & {$[-\mathrm{x}+2,-\mathrm{y}+1,-\mathrm{z}+1]$} \\
\hline N2-H4N2 & 0.900 & 2.130 & 147.77 & 2.931 & O19 & {$[\mathrm{x}+1 / 2,-\mathrm{y}+3 / 2, \mathrm{z}-1 / 2]$} \\
\hline $\mathrm{N} 2-\mathrm{H} 1 \mathrm{~N} 2$ & 0.900 & 2.107 & 150.51 & 2.925 & $\mathrm{O} 38$ & {$[\mathrm{x}+1, \mathrm{y}, \mathrm{z}]$} \\
\hline N3-H1N3 & 0.900 & 1.848 & 164.90 & 2.727 & $\mathrm{O} 21$ & {$[-\mathrm{x}+3 / 2, \mathrm{y}-1 / 2,-\mathrm{z}+3 / 2]$} \\
\hline N3-H3N3 & 0.900 & 1.952 & 157.88 & 2.806 & $\mathrm{O} 12$ & {$[-\mathrm{x}+1,-\mathrm{y}+1,-\mathrm{z}+1]$} \\
\hline N3-H4N3 & 0.900 & 1.886 & 162.86 & 2.759 & $\mathrm{O} 23$ & \\
\hline N4-H1N4 & 0.900 & 2.140 & 165.00 & 3.019 & O36 & \\
\hline N4-H3N4 & 0.900 & 2.209 & 129.81 & 2.870 & $\mathrm{O} 10$ & {$[\mathrm{x}-1, \mathrm{y}, \mathrm{z}]$} \\
\hline N4-H4N4 & 0.900 & 2.570 & 135.27 & 3.271 & $\mathrm{O} 15$ & {$[-\mathrm{x}+1,-\mathrm{y}+1,-\mathrm{z}+1]$} \\
\hline N4-H4N4 & 0.900 & 2.587 & 140.11 & 3.328 & $\mathrm{O} 12$ & \\
\hline
\end{tabular}


Table S4. Metric parameters $\left(\AA,^{\circ}\right)$ of $\left(\mathrm{Mo}_{7} \mathrm{O}_{24}\right)^{6-}$ unit in $\left(\mathrm{NH}_{4}\right)_{3}\left[\mathrm{Li}_{3}\left(\mathrm{H}_{2} \mathrm{O}\right)_{4}\left(\mu_{6}-\mathrm{Mo}_{7} \mathrm{O}_{24}\right)\right] \cdot 2 \mathrm{H}_{2} \mathrm{O} 2$.

\section{Bond lengths}

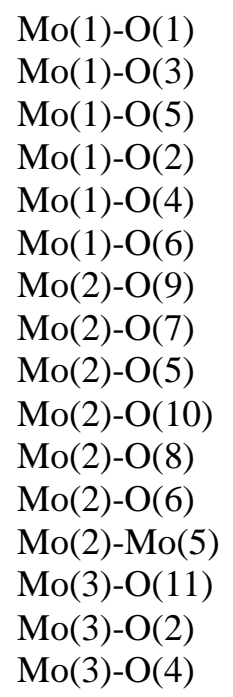

\section{Bond angles}

$\mathrm{O}(1)-\mathrm{Mo}(1)-\mathrm{O}(3)$ $\mathrm{O}(1)-\mathrm{Mo}(1)-\mathrm{O}(5)$ $\mathrm{O}(3)-\mathrm{Mo}(1)-\mathrm{O}(5)$ $\mathrm{O}(1)-\mathrm{Mo}(1)-\mathrm{O}(2)$ $\mathrm{O}(3)-\mathrm{Mo}(1)-\mathrm{O}(2)$ $\mathrm{O}(5)-\mathrm{Mo}(1)-\mathrm{O}(2)$ $\mathrm{O}(1)-\mathrm{Mo}(1)-\mathrm{O}(4)$ $\mathrm{O}(3)-\mathrm{Mo}(1)-\mathrm{O}(4)$ $\mathrm{O}(5)-\mathrm{Mo}(1)-\mathrm{O}(4)$ $\mathrm{O}(2)-\mathrm{Mo}(1)-\mathrm{O}(4)$ $\mathrm{O}(1)-\mathrm{Mo}(1)-\mathrm{O}(6)$ $\mathrm{O}(3)-\mathrm{Mo}(1)-\mathrm{O}(6)$ $\mathrm{O}(5)-\mathrm{Mo}(1)-\mathrm{O}(6)$ $\mathrm{O}(2)-\mathrm{Mo}(1)-\mathrm{O}(6)$ $\mathrm{O}(4)-\mathrm{Mo}(1)-\mathrm{O}(6)$ $\mathrm{O}(1)-\mathrm{Mo}(1)-\mathrm{Mo}(3)$ $\mathrm{O}(3)-\mathrm{Mo}(1)-\mathrm{Mo}(3)$ $\mathrm{O}(5)-\mathrm{Mo}(1)-\mathrm{Mo}(3)$ $\mathrm{O}(2)-\mathrm{Mo}(1)-\mathrm{Mo}(3)$ $\mathrm{O}(4)-\mathrm{Mo}(1)-\mathrm{Mo}(3)$ $\mathrm{O}(6)-\mathrm{Mo}(1)-\mathrm{Mo}(3)$ $\mathrm{O}(9)-\mathrm{Mo}(2)-\mathrm{O}(7)$ $\mathrm{O}(9)-\mathrm{Mo}(2)-\mathrm{O}(5)$ $\mathrm{O}(7)-\mathrm{Mo}(2)-\mathrm{O}(5)$ $\mathrm{O}(9)-\mathrm{Mo}(2)-\mathrm{O}(10)$ $\mathrm{O}(7)-\mathrm{Mo}(2)-\mathrm{O}(10)$ $\mathrm{O}(5)-\mathrm{Mo}(2)-\mathrm{O}(10)$ $\mathrm{O}(9)-\mathrm{Mo}(2)-\mathrm{O}(8)$

$\begin{array}{lll}1.720(3) & \mathrm{Mo}(3)-\mathrm{O}(13) & 2.477(4) \\ 1.721(3) & \mathrm{Mo}(3)-\mathrm{O}(12) & 1.753(4) \\ 1.939(2) & \mathrm{Mo}(3)-\mathrm{O}(2) \# 1 & 1.919(3) \\ 1.965(2) & \mathrm{Mo}(4)-\mathrm{O}(14) & 1.735(4) \\ 2.2207(7) & \mathrm{Mo}(4)-\mathrm{O}(13) & 1.745(4) \\ 2.237(2) & \mathrm{Mo}(4)-\mathrm{O}(6) \# 1 & 1.900(3) \\ 1.723(3) & \mathrm{Mo}(4)-\mathrm{O}(6) & 1.900(3) \\ 1.725(3) & \mathrm{Mo}(4)-\mathrm{O}(8) & 2.259(3) \\ 1.932(2) & \mathrm{Mo}(4)-\mathrm{O}(4) & 2.280(3) \\ 1.972(2) & \mathrm{Mo}(5)-\mathrm{O}(15) & 1.709(4) \\ 2.1457(9) & \mathrm{Mo}(5)-\mathrm{O}(16) & 1.748(4) \\ 2.249(2) & \mathrm{Mo}(5)-\mathrm{O}(10) & 1.907(3) \\ 3.1851(4) & \mathrm{Mo}(5)-\mathrm{O}(10) \# 1 & 1.907(3) \\ 1.715(4) & \mathrm{Mo}(5)-\mathrm{O}(8) & 2.158(3) \\ 1.919(3) & \mathrm{Mo}(2) \# 1-\mathrm{O}(8) & 2.1457(9) \\ 2.118(3) & & \end{array}$

105.81(17) 99.64(8) 99.60(8) 99.64(8) 99.60(8) 147.82(15) 151.62(18) 95.48(9) 101.56(9) 101.56(9) 102.22(14) $110.36(12)$ 105.04(16) 78.77(8) 71.45(12) 91.45(10) 134.23(4) 34.93(7) $119.22(7)$ 43.669(17) 78.77(8) 83.11(6) 91.45(10) 134.23(4) 119.22(7) 34.93(7) 43.669(17) 83.11(6) 


\begin{tabular}{|c|c|c|c|}
\hline $\mathrm{O}(7)-\mathrm{Mo}(2)-\mathrm{O}(8)$ & $158.31(13)$ & $\mathrm{O}(14)-\mathrm{Mo}(4)-\mathrm{O}(13)$ & $105.55(17)$ \\
\hline $\mathrm{O}(5)-\mathrm{Mo}(2)-\mathrm{O}(8)$ & $88.35(12)$ & $\mathrm{O}(14)-\mathrm{Mo}(4)-\mathrm{O}(6) \# 1$ & $100.34(8)$ \\
\hline $\mathrm{O}(10)-\mathrm{Mo}(2)-\mathrm{O}(8)$ & 73.04(12) & $\mathrm{O}(13)-\mathrm{Mo}(4)-\mathrm{O}(6) \# 1$ & $101.77(8)$ \\
\hline $\mathrm{O}(9)-\mathrm{Mo}(2)-\mathrm{O}(6)$ & $160.14(12)$ & $\mathrm{O}(14)-\mathrm{Mo}(4)-\mathrm{O}(6)$ & $100.34(8)$ \\
\hline $\mathrm{O}(7)-\mathrm{Mo}(2)-\mathrm{O}(6)$ & $91.45(11)$ & $\mathrm{O}(13)-\mathrm{Mo}(4)-\mathrm{O}(6)$ & $101.77(8)$ \\
\hline $\mathrm{O}(5)-\mathrm{Mo}(2)-\mathrm{O}(6)$ & $73.84(9)$ & $\mathrm{O}(6) \# 1-\mathrm{Mo}(4)-\mathrm{O}(6)$ & $143.00(14)$ \\
\hline $\mathrm{O}(10)-\mathrm{Mo}(2)-\mathrm{O}(6)$ & $83.60(10)$ & $\mathrm{O}(14)-\mathrm{Mo}(4)-\mathrm{O}(8)$ & $84.11(15)$ \\
\hline $\mathrm{O}(8)-\mathrm{Mo}(2)-\mathrm{O}(6)$ & $71.74(11)$ & $\mathrm{O}(13)-\mathrm{Mo}(4)-\mathrm{O}(8)$ & $170.33(15)$ \\
\hline $\mathrm{O}(9)-\mathrm{Mo}(2)-\mathrm{Mo}(5)$ & $86.49(10)$ & $\mathrm{O}(6) \# 1-\mathrm{Mo}(4)-\mathrm{O}(8)$ & $76.00(7)$ \\
\hline $\mathrm{O}(7)-\mathrm{Mo}(2)-\mathrm{Mo}(5)$ & $125.87(9)$ & $\mathrm{O}(6)-\mathrm{Mo}(4)-\mathrm{O}(8)$ & $76.00(7)$ \\
\hline $\mathrm{O}(5)-\mathrm{Mo}(2)-\mathrm{Mo}(5)$ & $130.75(8)$ & $\mathrm{O}(14)-\mathrm{Mo}(4)-\mathrm{O}(4)$ & $171.36(15)$ \\
\hline $\mathrm{O}(10)-\mathrm{Mo}(2)-\mathrm{Mo}(5)$ & $34.15(8)$ & $\mathrm{O}(13)-\mathrm{Mo}(4)-\mathrm{O}(4)$ & $83.09(14)$ \\
\hline $\mathrm{O}(8)-\mathrm{Mo}(2)-\mathrm{Mo}(5)$ & $42.41(9)$ & $\mathrm{O}(6) \# 1-\mathrm{Mo}(4)-\mathrm{O}(4)$ & $77.48(7)$ \\
\hline $\mathrm{O}(6)-\mathrm{Mo}(2)-\mathrm{Mo}(5)$ & $87.53(6)$ & $\mathrm{O}(6)-\mathrm{Mo}(4)-\mathrm{O}(4)$ & $77.48(7)$ \\
\hline $\mathrm{O}(11)-\mathrm{Mo}(3)-\mathrm{O}(4)$ & $101.10(16)$ & $\mathrm{O}(8)-\mathrm{Mo}(4)-\mathrm{O}(4)$ & $87.25(12)$ \\
\hline $\mathrm{O}(12)-\mathrm{Mo}(3)-\mathrm{O}(4)$ & $153.09(15)$ & $\mathrm{O}(15)-\mathrm{Mo}(5)-\mathrm{O}(16)$ & $106.44(17)$ \\
\hline $\mathrm{O}(2) \# 1-\mathrm{Mo}(3)-\mathrm{O}(4)$ & $75.59(7)$ & $\mathrm{O}(15)-\mathrm{Mo}(5)-\mathrm{O}(10)$ & $99.06(9)$ \\
\hline $\mathrm{O}(2)-\mathrm{Mo}(3)-\mathrm{O}(4)$ & $75.59(7)$ & $\mathrm{O}(16)-\mathrm{Mo}(5)-\mathrm{O}(10)$ & $101.04(8)$ \\
\hline $\mathrm{O}(11)-\mathrm{Mo}(3)-\mathrm{O}(13)$ & $172.55(16)$ & $\mathrm{O}(15)-\mathrm{Mo}(5)-\mathrm{O}(10) \# 1$ & $99.06(8)$ \\
\hline $\mathrm{O}(12)-\mathrm{Mo}(3)-\mathrm{O}(13)$ & $81.64(14)$ & $\mathrm{O}(16)-\mathrm{Mo}(5)-\mathrm{O}(10) \# 1$ & $101.04(8)$ \\
\hline $\mathrm{O}(15)-\mathrm{Mo}(5)-\mathrm{Mo}(2) \# 1$ & $91.21(10)$ & $\mathrm{O}(10)-\mathrm{Mo}(5)-\mathrm{Mo}(2) \# 1$ & $115.79(7)$ \\
\hline $\mathrm{O}(16)-\mathrm{Mo}(5)-\mathrm{Mo}(2) \# 1$ & $136.06(4)$ & $\mathrm{O}(8)-\mathrm{Mo}(5)-\mathrm{Mo}(2) \# 1$ & $42.11(2)$ \\
\hline
\end{tabular}

Table S5. Geometric parameters of the $\mathrm{N}-\mathrm{H} \cdots \mathrm{O}$ and $\mathrm{O}-\mathrm{H} \cdots \mathrm{O}$, hydrogen bonds length $(\AA)$ and $\left(^{\circ}\right)$ in the crystal structure of $\left(\mathrm{NH}_{4}\right)_{3}\left[\mathrm{Li}_{3}\left(\mathrm{H}_{2} \mathrm{O}\right)_{4}\left(\mu_{6}-\mathrm{Mo}_{7} \mathrm{O}_{24}\right)\right] \cdot 2 \mathrm{H}_{2} \mathrm{O} 2$.

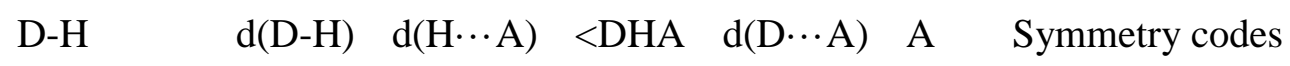

\begin{tabular}{lllllll}
\hline & & & & & & \\
N1-H1N1 & 0.840 & 2.049 & 178.60 & 2.889 & O16 & \\
N1-H2N1 & 0.840 & 2.361 & 134.82 & 3.012 & O3 & {$[-\mathrm{x}+1 / 2,-\mathrm{y}+1, \mathrm{z}-1 / 2]$} \\
N1-H2N1 & 0.840 & 2.361 & 134.82 & 3.012 & O3 & {$[-\mathrm{x}+1 / 2, \mathrm{y}-1 / 2, \mathrm{z}-1 / 2]$} \\
N1-H3N1 & 0.840 & 2.018 & 163.48 & 2.833 & O5 & {$[\mathrm{x}-1 / 2,-\mathrm{y}+1 / 2,-\mathrm{z}+3 / 2]$} \\
N2-H1N2 & 0.840 & 2.146 & 168.02 & 2.973 & O9 & {$[-\mathrm{x}+1, \mathrm{y}-1 / 2,-\mathrm{z}+1]$} \\
N2-H2N2 & 0.840 & 2.175 & 130.25 & 2.792 & O10 & {$[-\mathrm{x}+1 / 2, \mathrm{y}-1 / 2, \mathrm{z}-1 / 2]$} \\
N2-H3N2 & 0.840 & 2.292 & 141.54 & 2.995 & O11 & {$[\mathrm{x}, \mathrm{y}, \mathrm{z}-1]$} \\
N2-H3N2 & 0.840 & 2.362 & 123.42 & 2.910 & $\mathrm{O} 1$ & {$[\mathrm{x},-\mathrm{y}+1 / 2, \mathrm{z}-1]$} \\
N2-H4N2 & 0.840 & 2.182 & 148.31 & 2.930 & $\mathrm{O} 12$ & {$[\mathrm{x}+1 / 2, \mathrm{y},-\mathrm{z}+3 / 2]$} \\
O21-H21A & 0.880 & 1.998 & 144.79 & 2.764 & $\mathrm{O} 2$ & {$[-\mathrm{x}+1 / 2,-\mathrm{y}+1, \mathrm{z}-1 / 2]$} \\
O21-H21B & 0.880 & 2.470 & 121.86 & 3.028 & $\mathrm{O} 14$ & {$[\mathrm{x}+1 / 2, \mathrm{y},-\mathrm{z}+3 / 2]$} \\
O22-H22A & 0.880 & 1.865 & 176.60 & 2.744 & $\mathrm{O} 1$ & {$[-\mathrm{x}+1, \mathrm{y}+1 / 2,-\mathrm{z}+2]$} \\
\hline
\end{tabular}



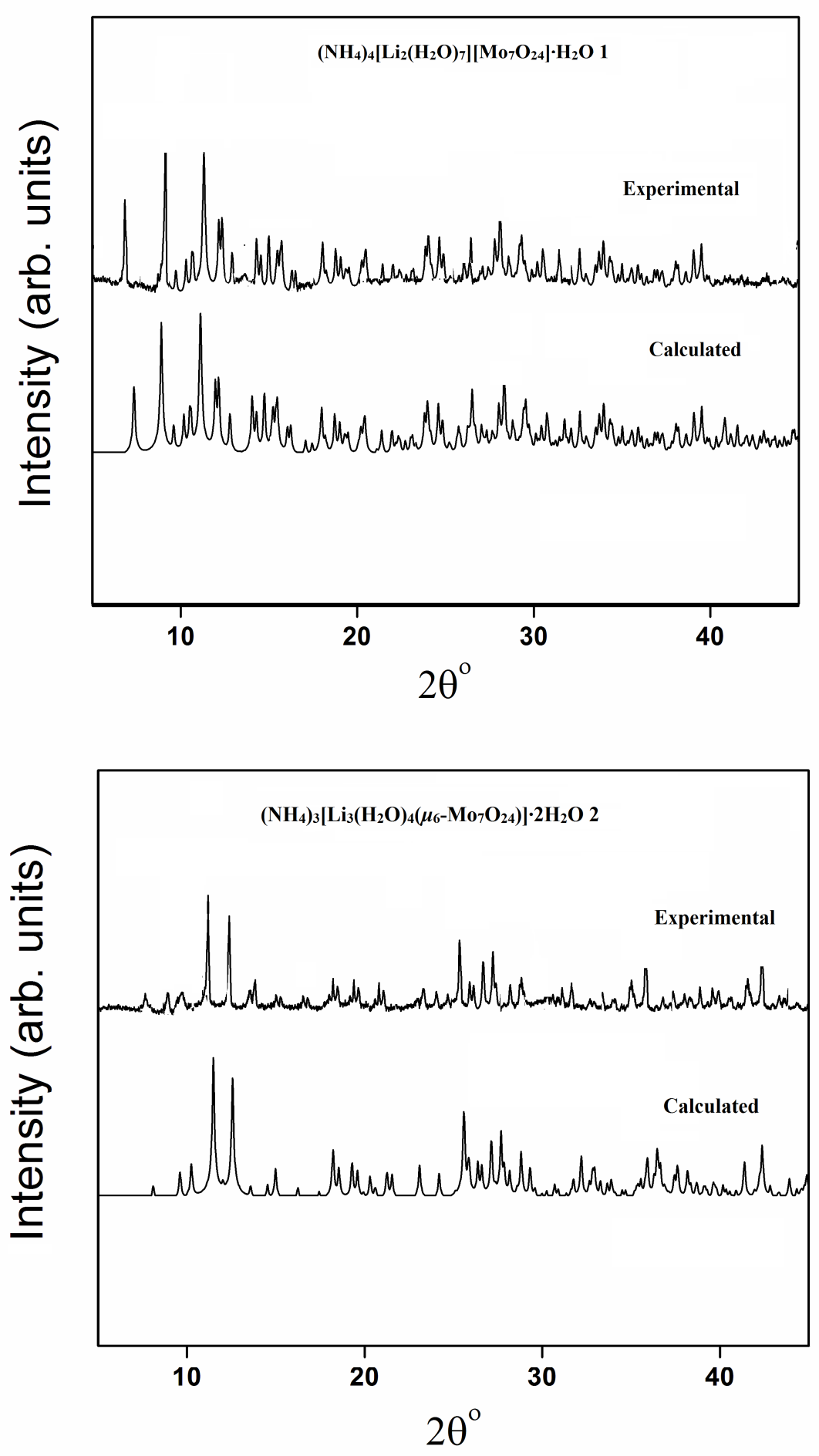

Figure S1. Calculated and experimental powder patterns of 1(top) \& 2 (bottom). 


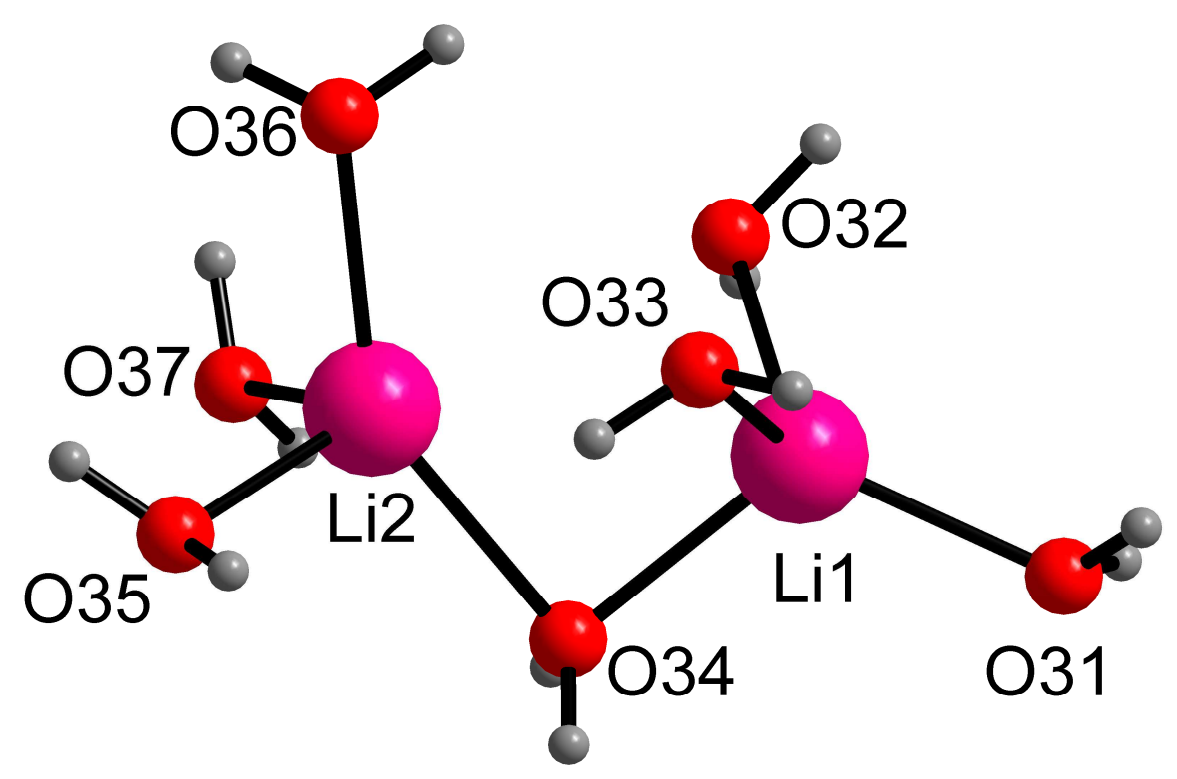

Figure S2. Coordination sphere of $\left\{\mathrm{LiO}_{4}\right\}$ tetrahedra around $\mathrm{Li} 1$ and $\mathrm{Li} 2$ showing $\mu_{2}$-bridging bidentate coordination of $\mathrm{O} 34$ resulting in a water bridged dinuclear cationic unit $\left[\mathrm{Li}_{2}\left(\mathrm{H}_{2} \mathrm{O}\right)_{7}\right]^{2+}$ in $\mathbf{1}$.
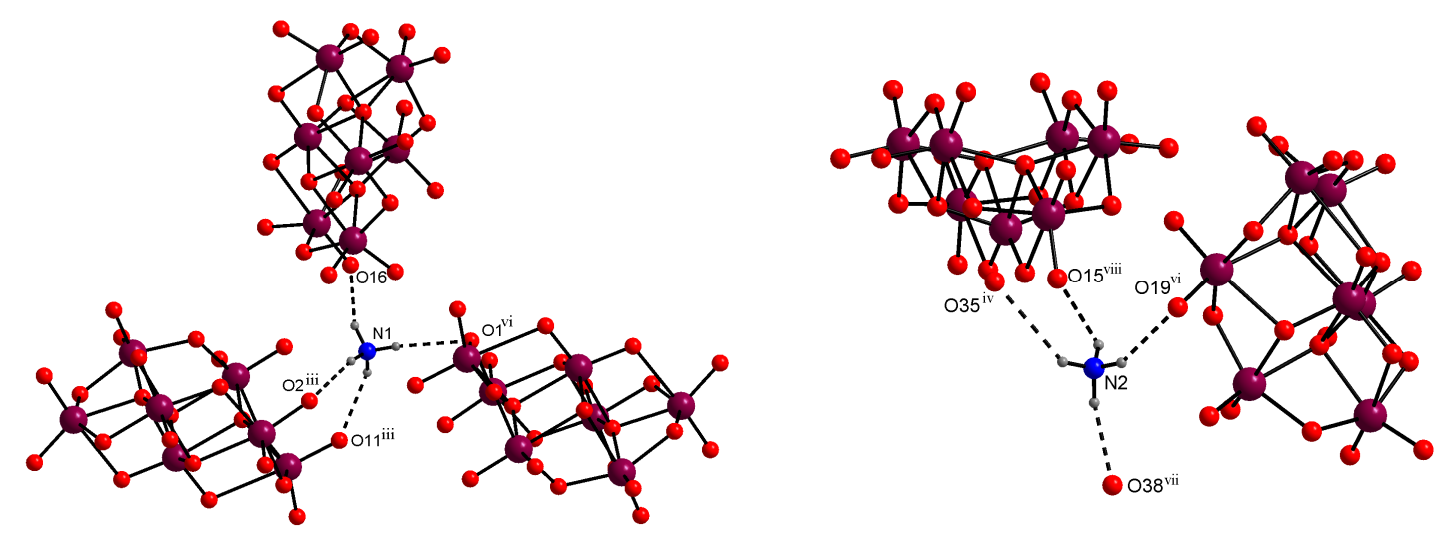

Figure S3. The hydrogen bonding situation around ammonium cations 'N1' (left) and 'N2' (right) showing intramolecular and intermolecular $\mathrm{N}-\mathrm{H} \cdots \mathrm{O}$ interactions (black dotted lines). Symmetry codes: iii) 3/2-x, 1/2+y, $3 / 2$ - iv) $1-\mathrm{x}, 1-\mathrm{y}, 1-\mathrm{z}$ vi) $-1 / 2+\mathrm{x}, 3 / 2-\mathrm{y}, 1 / 2+\mathrm{z}$ vii) $1+\mathrm{x}, \mathrm{y}, \mathrm{z}$ viii) $1-\mathrm{x}, 1-\mathrm{y}, 1-\mathrm{z}$. 

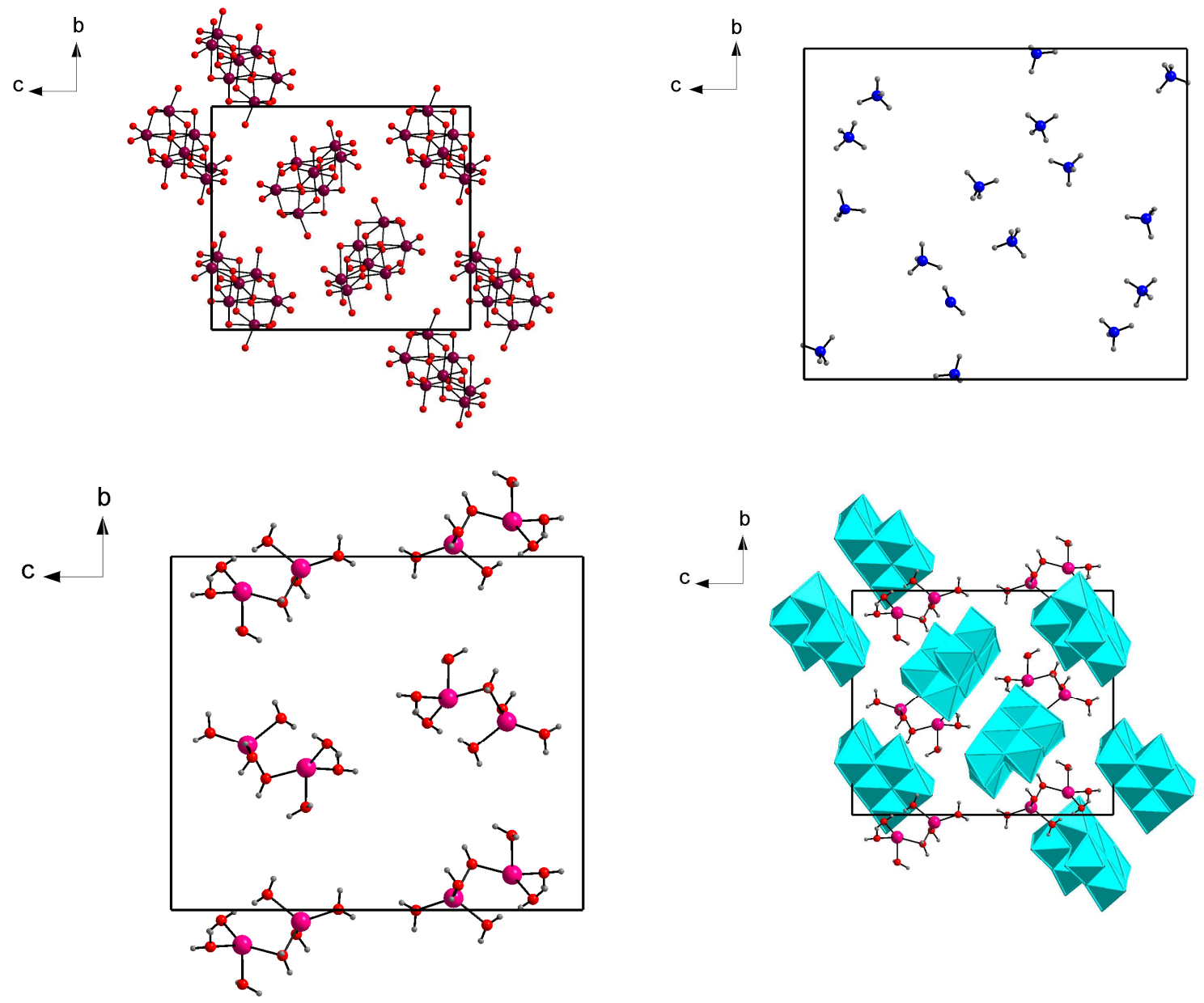

Figure S4. A view along ' $a$ ' axis of the unit cell packing showing only heptamolybdate anions (top left), ammonium cations (top right), $\left[\mathrm{Li}_{2}\left(\mathrm{H}_{2} \mathrm{O}\right)_{7}\right]^{2+}$ cationic unit (bottom left) and $\left[\mathrm{Li}_{2}\left(\mathrm{H}_{2} \mathrm{O}\right)_{7}\right]\left[\mathrm{Mo}_{7} \mathrm{O}_{24}\right]^{4-}$ unit (bottom right) in compound 1.Colour codes: Mo, maroon; Li, pink; O, red; N, blue; H, medium grey; heptamolybdate anions are shown as polyhedra.
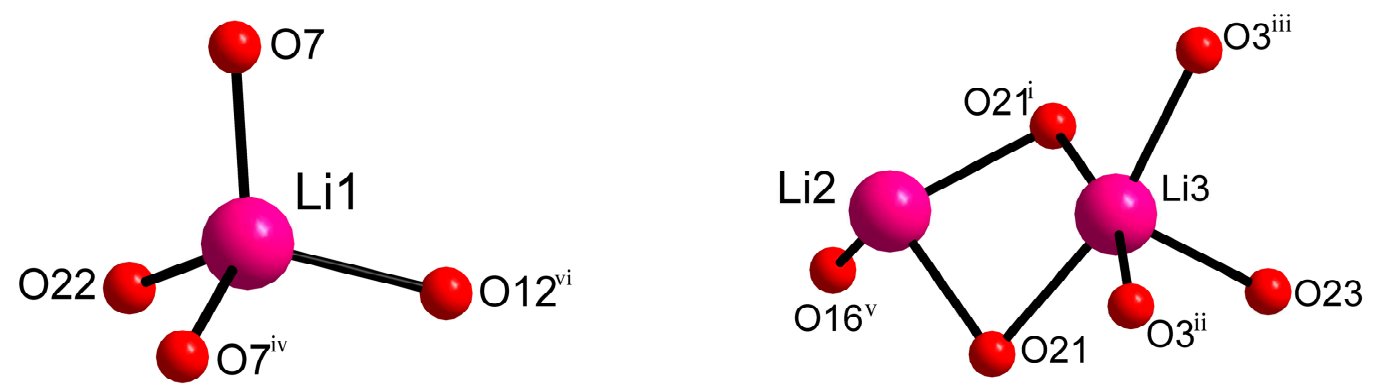

Figure S5. The coordination sphere of Li1 (left) and Li2, Li3 (right) in compound 2. Symmetry codes: i) $x$, $1 / 2-y, z$ ii) $x, 3 / 2-y, z$ iii) $-1 / 2+x, 1 / 2-y, 3 / 2-z$ iv) $1 / 2-x, 1-y,-1 / 2+z$ v) $1 / 2-x,-1 / 2+y,-1 / 2+z$ vi) $-1 / 2+x, 3 / 2-y$, $1 / 2+\mathrm{z}$ 

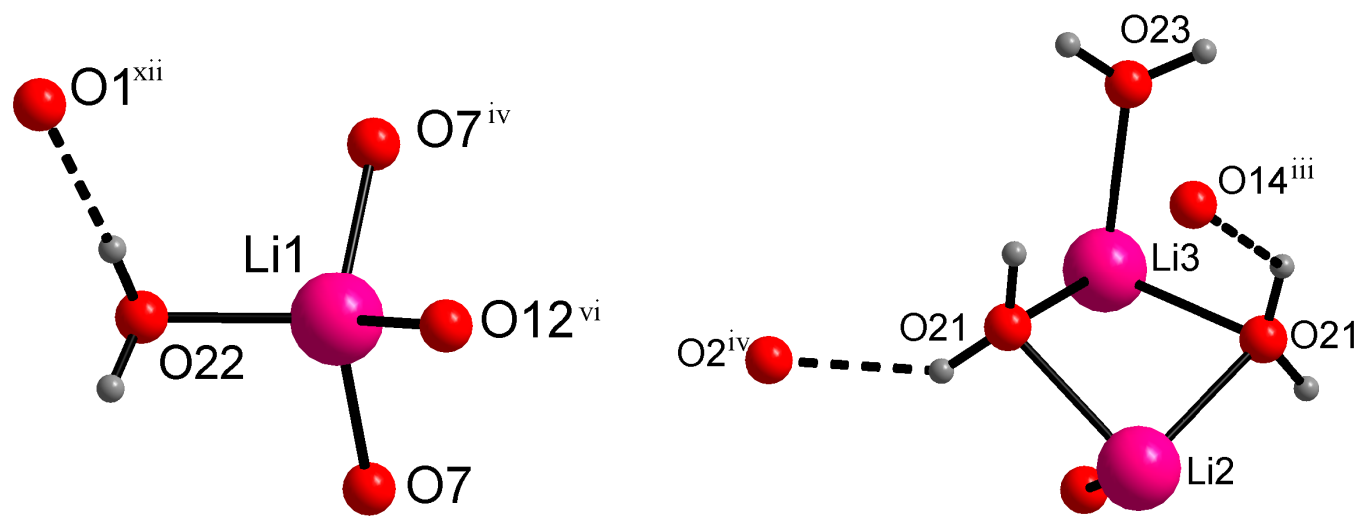

Figure S6. The hydrogen bonding situation around $\mathrm{Li} 1$ (left) and $\mathrm{Li} 2$, $\mathrm{Li} 3$ (right) showing $\mathrm{O}-\mathrm{H} \cdots \mathrm{O}$ interactions (black dotted lines). Symmetry codes: iii) $-1 / 2+\mathrm{x}, 1 / 2-\mathrm{y}, 3 / 2-\mathrm{z}$ iv) $1 / 2-\mathrm{x}, 1-\mathrm{y},-1 / 2+\mathrm{z}$ vi) $1 / 2-\mathrm{x}, 1 / 2+\mathrm{y}, 1 / 2+\mathrm{z}$ xii) $1-\mathrm{x},-1 / 2+\mathrm{y}, 2-\mathrm{z}$.
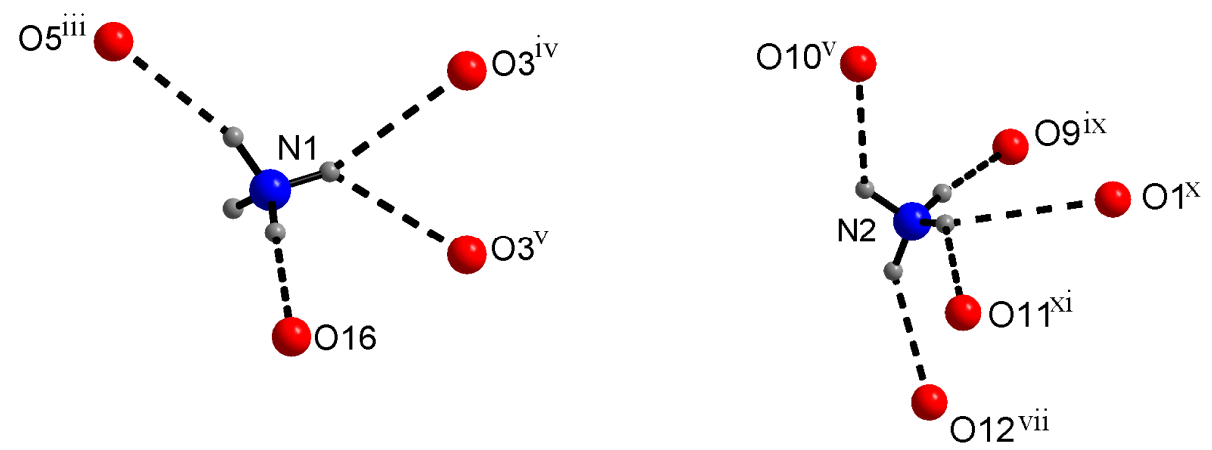

Figure S7. The hydrogen bonding situation around ammonium cations 'N1' (left) and 'N2' (right) shown by black dotted lines. Symmetry codes: iii) $-1 / 2+x, 1 / 2-y, 3 / 2-z$ iv) $1 / 2-x, 1-y,-1 / 2+z$ v) $1 / 2-x,-1 / 2+y,-1 / 2+z$ vii) $1 / 2+x, y, 3 / 2-z$ ix) $1-x, 1 / 2+y, 1-z$ x) $x, 1 / 2-y,-1+z$ xi) $x, y, 1+z$. 


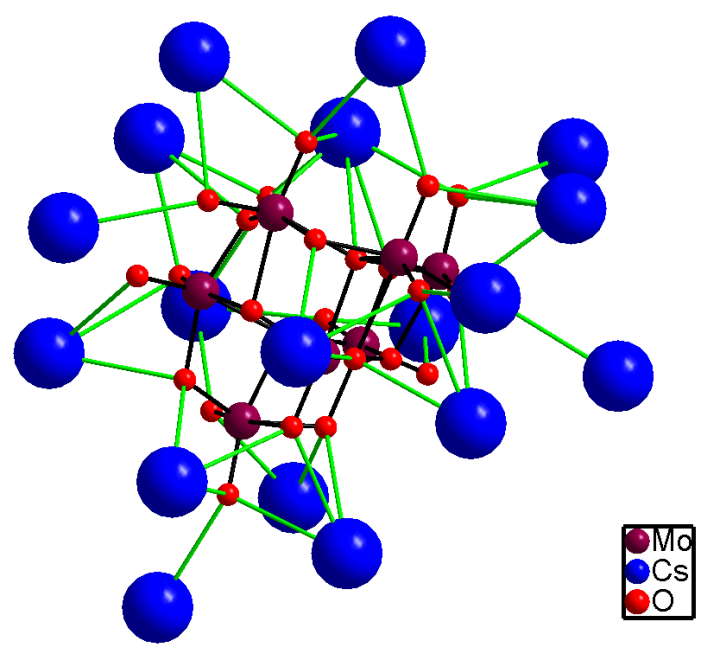

$\mu_{18}$-tritetracontadentate

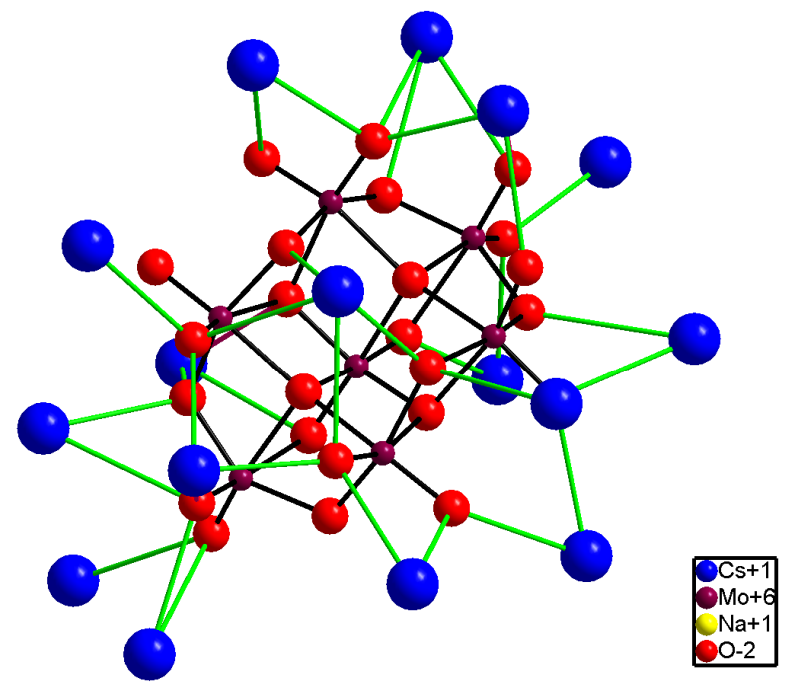

$\mu_{16}$-hentriacontadentate

Figure S8. The binding modes of heptamolybdate in $\mathrm{Cs}_{6}\left[\mathrm{Mo}_{7} \mathrm{O}_{24}\right] \cdot 7 \mathrm{H}_{2} \mathrm{O}$ (top), $\mathrm{NaCs}_{5}\left[\mathrm{Mo}_{7} \mathrm{O}_{24}\right] \cdot 5 \mathrm{H}_{2} \mathrm{O}$ (bottom) 

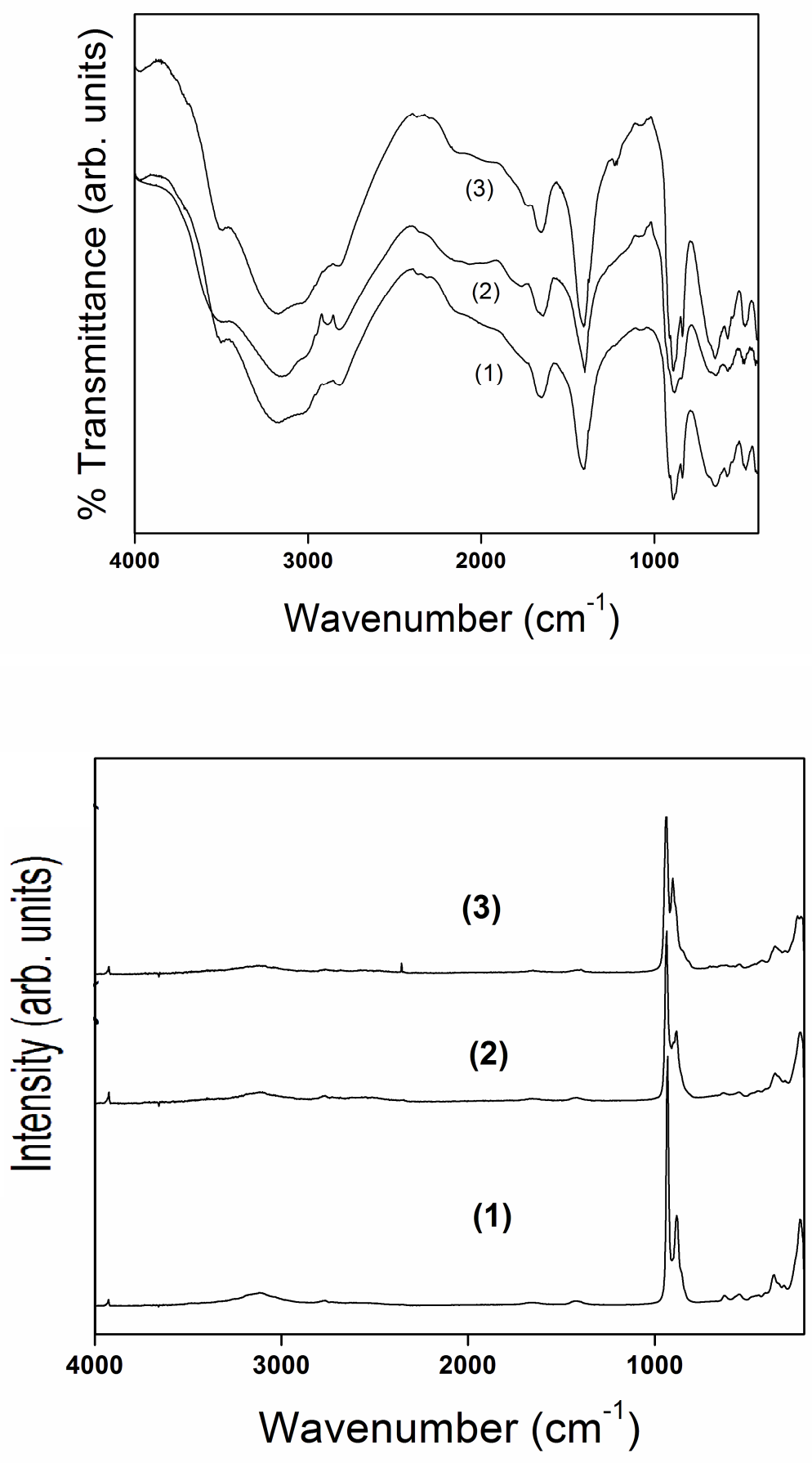

Figure S9. IR (top) and Raman spectra (bottom) of $\left(\mathrm{NH}_{4}\right)_{4}\left[\mathrm{Li}_{2}\left(\mathrm{H}_{2} \mathrm{O}\right)_{7}\right]\left[\mathrm{Mo}_{7} \mathrm{O}_{24}\right] \cdot \mathrm{H}_{2} \mathrm{O}(1)$, $\left(\mathrm{NH}_{4}\right)_{3}\left[\mathrm{Li}_{3}\left(\mathrm{H}_{2} \mathrm{O}\right)_{4}\left(\mu_{6}-\mathrm{Mo}_{7} \mathrm{O}_{24}\right)\right] \cdot 2 \mathrm{H}_{2} \mathrm{O}(2)$ and $\left(\mathrm{NH}_{4}\right)_{6}\left[\mathrm{Mo}_{7} \mathrm{O}_{24}\right] \cdot 4 \mathrm{H}_{2} \mathrm{O}$ (3). 

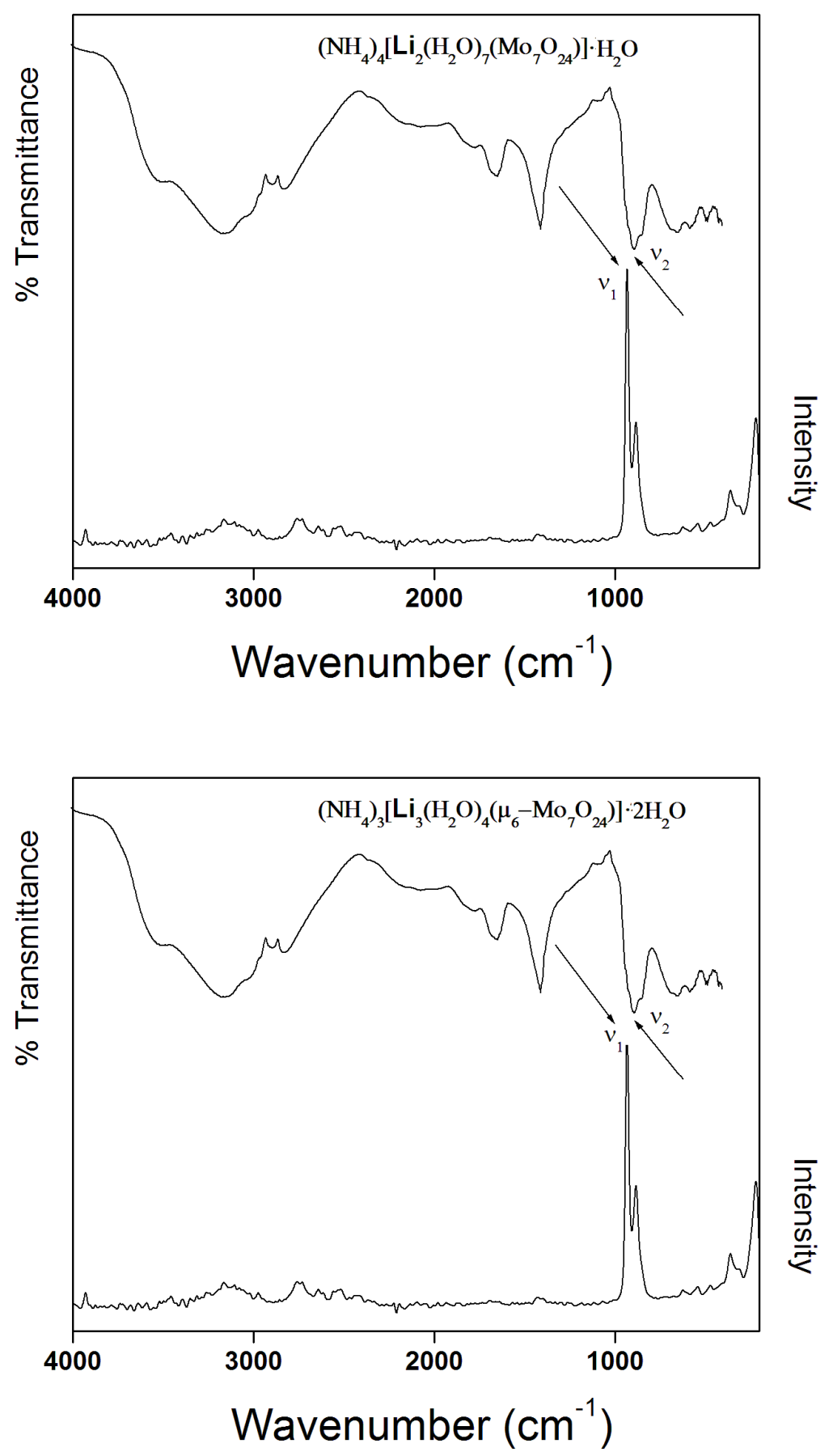

Figure S10. IR and Raman spectra of $\left(\mathrm{NH}_{4}\right)_{4}\left[\mathrm{Li}_{2}\left(\mathrm{H}_{2} \mathrm{O}\right)_{7}\right]\left[\mathrm{Mo}_{7} \mathrm{O}_{24}\right] \cdot \mathrm{H}_{2} \mathrm{O} \mathbf{1}$ (top) and $\left(\mathrm{NH}_{4}\right)_{3}\left[\mathrm{Li}_{3}\left(\mathrm{H}_{2} \mathrm{O}\right)_{4}\left(\mu_{6}-\mathrm{Mo}_{7} \mathrm{O}_{24}\right)\right] \cdot 2 \mathrm{H}_{2} \mathrm{O} 2$ (bottom). 


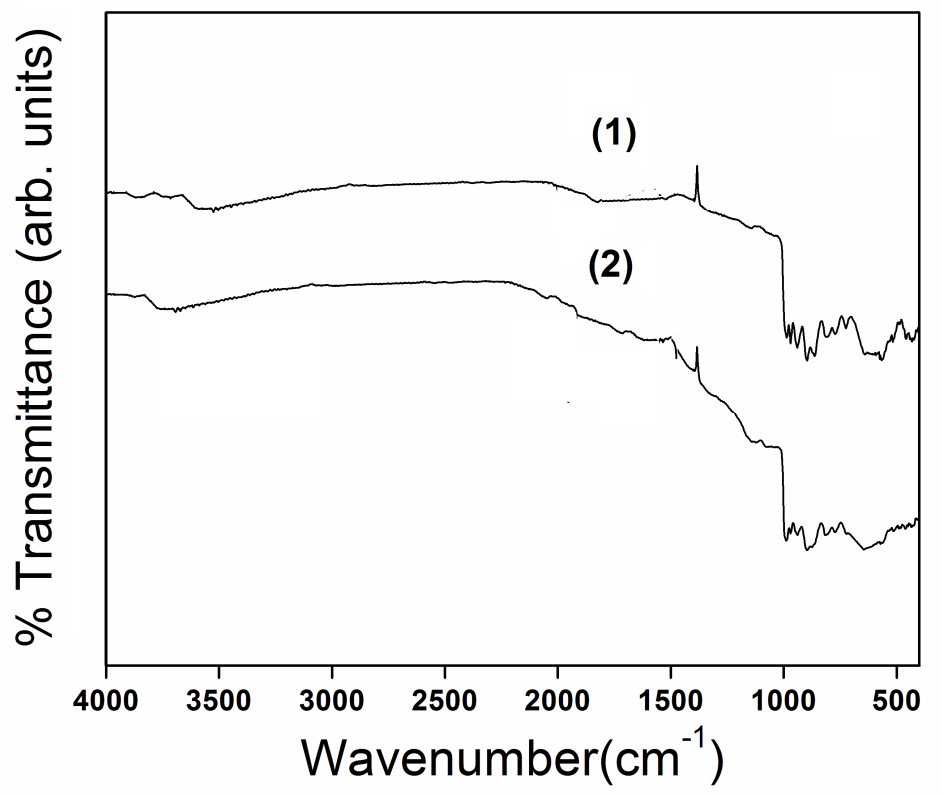

Figure S11. IR spectra of the residue obtained after heating $\left(\mathrm{NH}_{4}\right)_{4}\left[\mathrm{Li}_{2}\left(\mathrm{H}_{2} \mathrm{O}\right)_{7}\right]\left[\mathrm{Mo}_{7} \mathrm{O}_{24}\right] \cdot \mathrm{H}_{2} \mathrm{O}(1)$, $\left(\mathrm{NH}_{4}\right)_{3}\left[\mathrm{Li}_{3}\left(\mathrm{H}_{2} \mathrm{O}\right)_{4}\left(\mu_{6}-\mathrm{Mo}_{7} \mathrm{O}_{24}\right)\right] \cdot 2 \mathrm{H}_{2} \mathrm{O}(2)$ at $600{ }^{\circ} \mathrm{C}$.

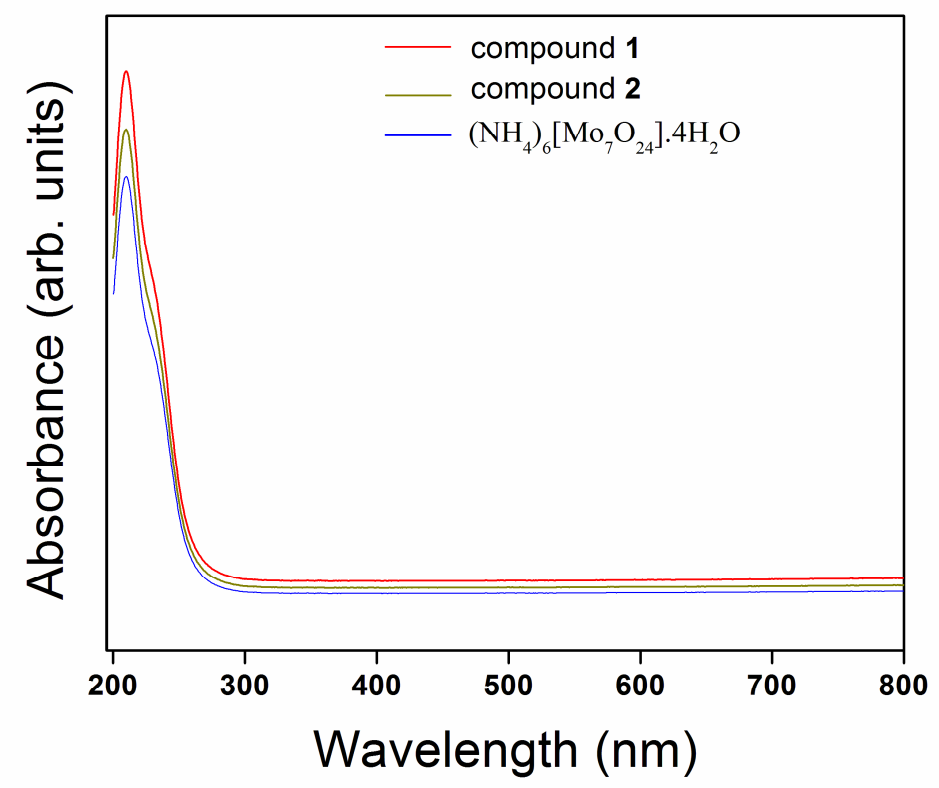

Figure S12. UV-Vis spectra of compound 1,2 and $\left(\mathrm{NH}_{4}\right)_{6}\left[\mathrm{Mo}_{7} \mathrm{O}_{24}\right] \cdot 4 \mathrm{H}_{2} \mathrm{O}$. 


\section{checkCIF/PLATON report $\left(\mathrm{NH}_{4}\right)_{4}\left[\mathrm{Li}_{2}\left(\mathrm{H}_{2} \mathrm{O}\right)_{7}\right]\left[\mathrm{Mo}_{7} \mathrm{O}_{24}\right] \cdot \mathrm{H}_{2} \mathrm{O} 1$}

You have not supplied any structure factors. As a result the full set of tests cannot be run.

THIS REPORT IS FOR GUIDANCE ONLY. IF USED AS PART OF A REVIEW PROCEDURE FOR PUBLICATION, IT SHOULD NOT REPLACE THE EXPERTISE OF AN EXPERIENCED CRYSTALLOGRAPHIC REFEREE.

No syntax errors found. CIF dictionary Interpreting this report

\section{Datablock: srini903}

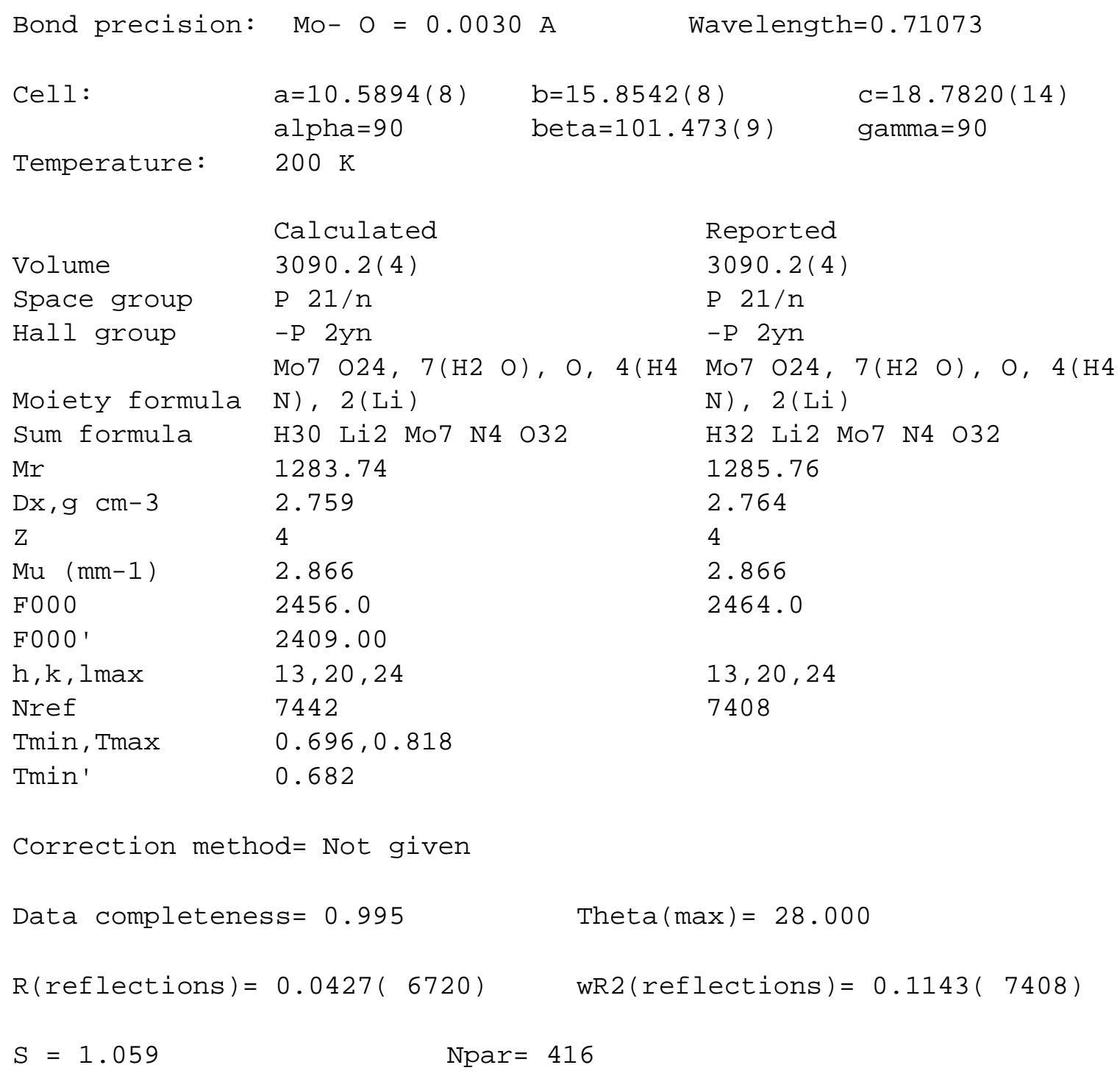


Alert level $B$

PLAT430_ALERT_2_B Short Inter D...A Contact PLAT430_ALERT_2_B Short Inter D...A Contact

011

011
. 038

. $038^{\prime}$
.

$\cdots$
2.74 Ang.

2.80 Ang.

\section{Alert level C}

PLAT041_ALERT_1_C Calc. and Reported SumFormula Strings Differ PLAT043_ALERT_1_C Calculated and Reported Mol. Weight Differ by .. PLAT052_ALERT_1_C Info on Absorption Correction Method Not Given PLAT057_ALERT_3_C Correction for Absorption Required RT (exp) ... PLAT068_ALERT_1_C Reported F000 Differs from Calcd (or Missing)...

Please Check 2.02 Check

Please Do ! 1.18 Do !

Please Check

\section{Alert level G}

FORMU01_ALERT_1_G There is a discrepancy between the atom counts in the _chemical_formula_sum and_chemical_formula_moiety. This is usually due to the moiety formula being in the wrong format. Atom count from_chemical_formula_sum: H32 Li2 Mo7 N4 032 Atom count from_chemical_formula_moiety:H30 Li2 Mo7 N4 032

FORMU01_ALERT_2_G There is a discrepancy between the atom counts in the _chemical_formula_sum and the formula from the _atom_site* data. Atom count from_chemical_formula_sum:H32 Li2 Mo7 N4 032 Atom count from the atom_site data: H30 Li2 Mo7 N4 032 CELLZ01_ALERT_1_G Difference between formula and atom_site contents detected. CELLZ01_ALERT_1_G WARNING: $\mathrm{H}$ atoms missing from atom site list. Is this intentional?

From the CIF:_cell_formula_units_z 4

From the CIF: _chemical_formula_sum H32 Li2 Mo7 N4 032

TEST: Compare cell contents of formula and atom_site data

$\begin{array}{lcrl}\text { atom } & \text { Z*formula } & \text { cif sites diff } \\ \mathrm{H} & 128.00 & 120.00 & 8.00 \\ \mathrm{Li} & 8.00 & 8.00 & 0.00 \\ \mathrm{Mo} & 28.00 & 28.00 & 0.00 \\ \mathrm{~N} & 16.00 & 16.00 & 0.00 \\ \mathrm{O} & 128.00 & 128.00 & 0.00\end{array}$

PLAT005_ALERT_5_G No Embedded Refinement Details found in the CIF

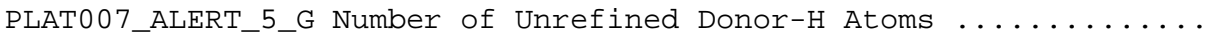
PLAT093_ALERT_1_G No s.u.'s on H-positions, Refinement Reported as PLAT232_ALERT_2_G Hirshfeld Test Diff (M-X) Mo5 - 014 .. PLAT300_ALERT_4_G Atom Site Occupancy of >038 is Constrained at PLAT300_ALERT_4_G Atom Site Occupancy of <038' is Constrained at PLAT302_ALERT_4_G Anion/Solvent Disorder ........ Percentage = PLAT304_ALERT_4_G Non-Integer Number of Atoms ( 0.75$)$ in Resd. \# PLAT304_ALERT_4_G Non-Integer Number of Atoms $(0.25)$ in Resd. \# PLAT311_ALERT_2_G Isolated Disordered Oxygen Atom (No H's ?) ..... PLAT311_ALERT_2_G Isolated Disordered Oxygen Atom (No H's ?) ..... PLAT720_ALERT_4_G Number of Unusual/Non-Standard Labels ........ PLAT899_ALERT_4_G SHELXL97 is Deprecated and Succeeded by SHELXL

Please Do ! 30 Report mixed Check $5.3 \mathrm{~s} . \mathrm{u}$. 0.75 Check 0.25 check

7 Note

9 Check

14 Check 038 Check 038 ' Check

16 Note 2014 Note

0 ALERT level $\mathbf{A}=$ Most likely a serious problem - resolve or explain 2

ALERT level B = A potentially serious problem, consider carefully

5 ALERT level $\mathbf{C}=$ Check. Ensure it is not caused by an omission or oversight 17

ALERT level $\mathbf{G}=$ General information/check it is not something unexpected
8 ALERT type 1 CIF construction/syntax error, inconsistent or missing data
6 ALERT type 2 Indicator that the structure model may be wrong or deficient
1 ALERT type 3 Indicator that the structure quality may be low
7 ALERT type 4 Improvement, methodology, query or suggestion
2 ALERT type 5 Informative message, check 


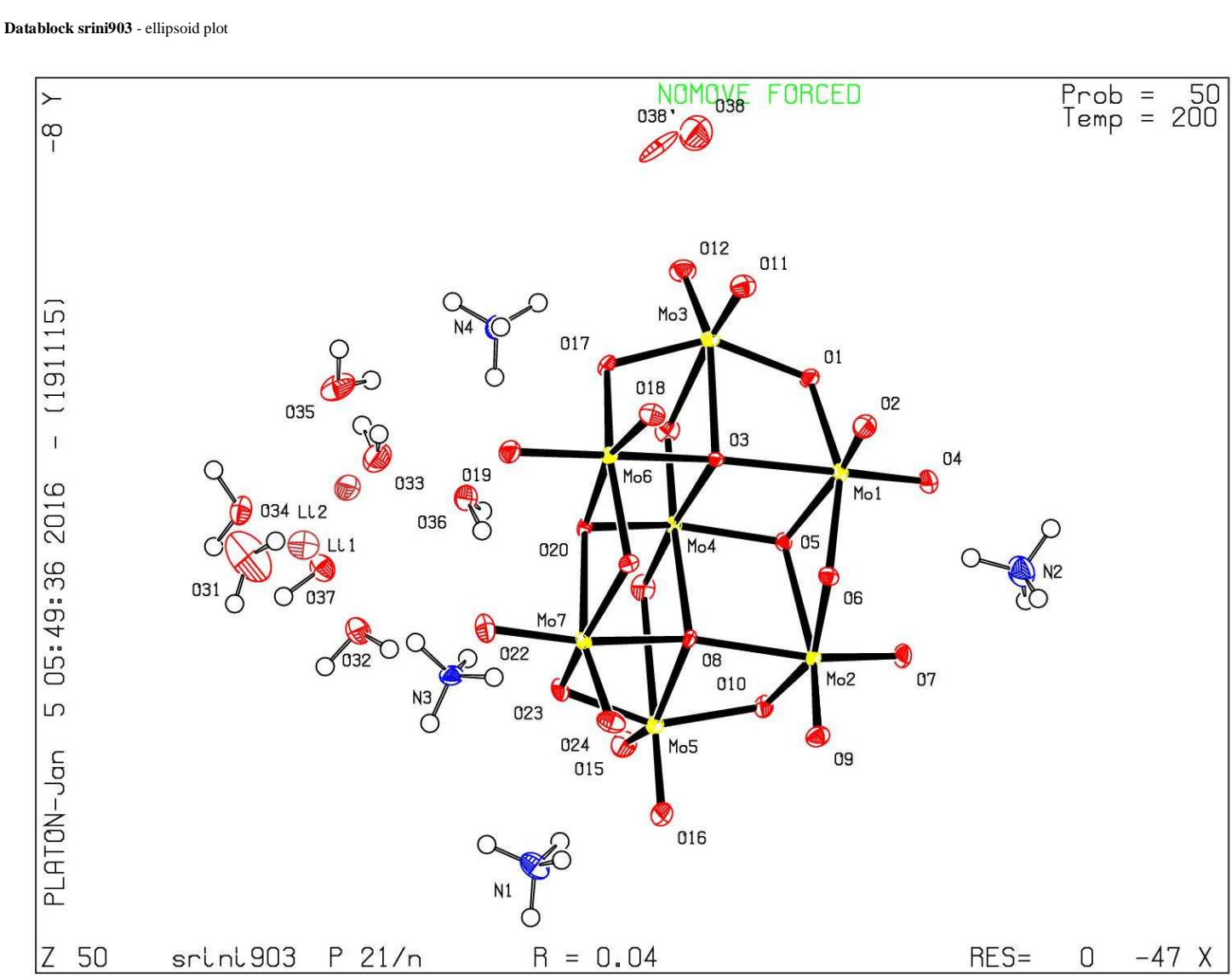




\section{checkCIF/PLATON report of $\left(\mathrm{NH}_{4}\right)_{3}\left[\mathrm{Li}_{3}\left(\mathrm{H}_{2} \mathrm{O}\right)_{4}\left(\mu_{6}-\mathrm{Mo}_{7} \mathrm{O}_{24}\right)\right] \cdot 2 \mathrm{H}_{2} \mathrm{O} 2$}

You have not supplied any structure factors. As a result the full set of tests cannot be run.

THIS REPORT IS FOR GUIDANCE ONLY. IF USED AS PART OF A REVIEW PROCEDURE FOR PUBLICATION, IT SHOULD NOT REPLACE THE EXPERTISE OF AN EXPERIENCED CRYSTALLOGRAPHIC REFEREE.

No syntax errors found. CIF dictionary Interpreting this report

\section{Datablock: srini903}

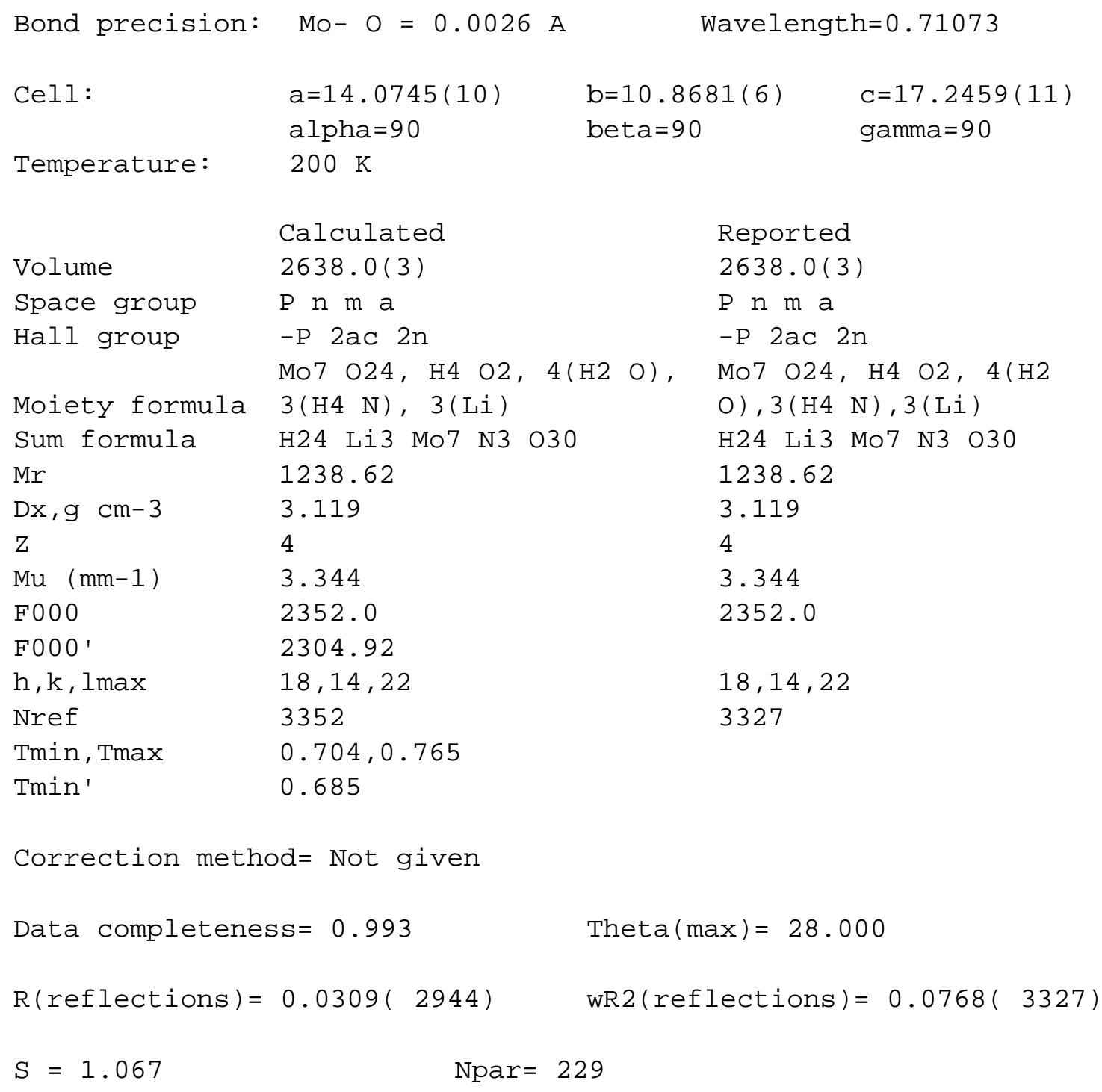


Alert level A

PLAT417_ALERT_2_A Short Inter D-H..H-D H23A $\quad$ H H24A $\quad$ H $\quad 1.35$ Ang.

\section{Alert level B}

PLAT213_ALERT_2_B Atom Mo4

has ADP max/min Ratio ......

4.2 oblate

\section{Alert level C}

PLAT052_ALERT_1_C Info on Absorption Correction Method Not Given PLAT220_ALERT_2_C Large Non-Solvent $O$ Ueq (max)/Ueq(min) Range

PLAT417_ALERT_2_C Short Inter D-H..H-D H21B .. H24A .

Please Do !

3.1 Ratio

2.14 Ang.

\section{Alert level G}

PLAT005_ALERT_5_G No Embedded Refinement Details found in the CIF PLAT007_ALERT_5_G Number of Unrefined Donor-H Atoms ........... PLAT042_ALERT_1_G Calc. and Reported MoietyFormula Strings Differ PLAT093_ALERT_1_G No s.u.'s on H-positions, Refinement Reported as PLAT300_ALERT_4_G Atom Site Occupancy of >024 is Constrained at PLAT300_ALERT_4_G Atom Site Occupancy of <O24' is Constrained at PLAT302_ALERT_4_G Anion/Solvent Disorder ........ Percentage = PLAT304_ALERT_4_G Non-Integer Number of Atoms ( 0.50$)$ in Resd. \# PLAT304_ALERT_4_G Non-Integer Number of Atoms ( 0.50$)$ in Resd. \# PLAT304_ALERT_4_G Non-Integer Number of Atoms $(0.50)$ in Resd. \# PLAT720_ALERT_4_G Number of Unusual/Non-Standard Labels ........ PLAT899_ALERT_4_G SHELXL97 is Deprecated and Succeeded by SHELXL
Please Do !
13 Report
Please Check mixed Check
0.75 Check
0.25 Check
20 Note
8 Check
9 Check
10 Check
7 Note
2014 Note

\footnotetext{
1 ALERT level $\mathbf{A}=$ Most likely a serious problem - resolve or explain 1

ALERT level B = A potentially serious problem, consider carefully

3 ALERT level $\mathbf{C}=$ Check. Ensure it is not caused by an omission or oversight

12 ALERT level $\mathbf{G}=$ General information/check it is not something unexpected

3 ALERT type 1 CIF construction/syntax error, inconsistent or missing data

4 ALERT type 2 Indicator that the structure model may be wrong or deficient

0 ALERT type 3 Indicator that the structure quality may be low

8 ALERT type 4 Improvement, methodology, query or suggestion

2 ALERT type 5 Informative message, check
} 
Datablock srini903 - ellipsoid plot

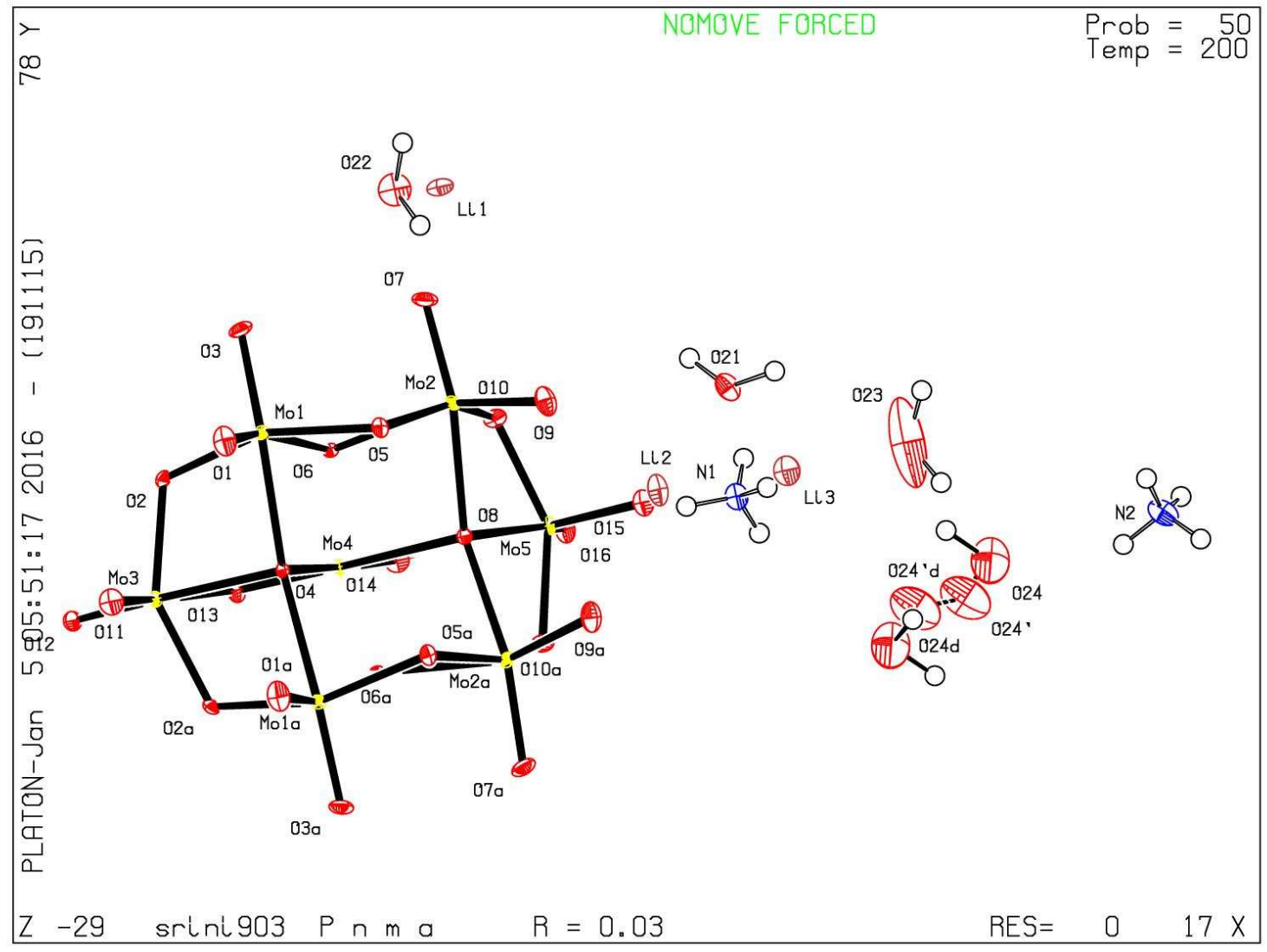

